\title{
Quantum-Classical Correspondence Principle for Work Distributions
}

\author{
Christopher Jarzynski, ${ }^{1, *}$ H. T. Quan, ${ }^{2,3, \dagger}$ and Saar Rahav, ${ }^{4,+}$ \\ ${ }^{1}$ Department of Chemistry and Biochemistry and Institute of Physical Science and Technology, \\ University of Maryland, College Park, Maryland 20742, USA \\ ${ }^{2}$ School of Physics, Peking University, Beijing 100871, China \\ ${ }^{3}$ Collaborative Innovation Center of Quantum Matter, Beijing 100871, China \\ ${ }^{4}$ Schulich Faculty of Chemistry, Technion-Israel Institute of Technology, Haifa 32000, Israel
}

(Received 14 May 2015; published 17 September 2015)

\begin{abstract}
For closed quantum systems driven away from equilibrium, work is often defined in terms of projective measurements of initial and final energies. This definition leads to statistical distributions of work that satisfy nonequilibrium work and fluctuation relations. While this two-point measurement definition of quantum work can be justified heuristically by appeal to the first law of thermodynamics, its relationship to the classical definition of work has not been carefully examined. In this paper, we employ semiclassical methods, combined with numerical simulations of a driven quartic oscillator, to study the correspondence between classical and quantal definitions of work in systems with 1 degree of freedom. We find that a semiclassical work distribution, built from classical trajectories that connect the initial and final energies, provides an excellent approximation to the quantum work distribution when the trajectories are assigned suitable phases and are allowed to interfere. Neglecting the interferences between trajectories reduces the distribution to that of the corresponding classical process. Hence, in the semiclassical limit, the quantum work distribution converges to the classical distribution, decorated by a quantum interference pattern. We also derive the form of the quantum work distribution at the boundary between classically allowed and forbidden regions, where this distribution tunnels into the forbidden region. Our results clarify how the correspondence principle applies in the context of quantum and classical work distributions and contribute to the understanding of work and nonequilibrium work relations in the quantum regime.
\end{abstract}

DOI: 10.1103/PhysRevX.5.031038

\section{INTRODUCTION}

Work is a familiar concept in elementary mechanics and a central one in thermodynamics. In recent years, interest in the nonequilibrium thermodynamics of small systems [1-4] has motivated careful examinations of how to define quantum work [5-50]. In this context one often considers a process in which a quantum system evolves under the Schrödinger equation as its Hamiltonian is varied in timefor instance, a quantum particle in a piston undergoing compression or expansion [51]. It is typically assumed that the system is initialized in thermal equilibrium, and the question becomes, how do we appropriately define the work performed on the system during a single realization of this process?

One answer involves two projective measurements of the system's energy, at the start of the process $(t=0)$ and at the

\footnotetext{
*cjarzyns@umd.edu

†htquan@pku.edu.cn

ॠrahavs@tx.technion.ac.il
}

Published by the American Physical Society under the terms of the Creative Commons Attribution 3.0 License. Further distribution of this work must maintain attribution to the author(s) and the published article's title, journal citation, and DOI.
Subject Areas: Quantum Physics, Statistical Physics

end $(t=\tau)$. If the system Hamiltonian is varied from $\hat{H}(0)=\hat{H}_{A}$ to $\hat{H}(\tau)=\hat{H}_{B}$, then the measurement outcomes will be eigenvalues of these operators. The work performed during the process is then defined to be the difference between these two values, e.g.,

$$
W=E_{n}^{B}-E_{m}^{A},
$$

if the measurements produce the $m$ th and $n$th eigenvalues of the initial and final Hamiltonians. In this definition, work is inherently stochastic, with two sources of randomness: the statistical randomness associated with sampling an initial energy $E_{m}^{A}$ from the canonical equilibrium distribution [Eq. (21)] and the purely quantal randomness associated with the "collapse" of the final wave function $\left|\psi_{\tau}\right\rangle$ upon making a projective measurement of the final energy [Eq. (22)] [52].

In an analogous classical process, a system is prepared in thermal equilibrium, then it evolves under Hamilton's equations as the Hamiltonian function is varied from $H_{A}(z)$ to $H_{B}(z)$, where $z$ denotes a point in the system's phase space. Since the system is thermally isolated, it is natural to define the work as the change in its internal energy: 


$$
W=H_{B}\left(z_{\tau}\right)-H_{A}\left(z_{0}\right),
$$

for a realization during which the system evolves from $z_{0}$ to $z_{\tau}$. As in the quantum case, $W$ is a stochastic variable, but here the randomness arises solely from the sampling of the initial microstate $z_{0}$ from an equilibrium distribution.

Much of the recent interest in quantum work has been stimulated by the discovery and experimental verification of classical nonequilibrium work relations [53-58], which have provided insights into the second law of thermodynamics, particularly as it applies to small systems where fluctuations are important [2]. For thermally isolated systems, the quantal counterparts of these relations follow directly from the definition of work given by Eq. (1) $[6,7,9,15]$. Moreover, experimental tests of quantum nonequilibrium work relations have recently been proposed [59-62] and implemented [63,64], providing direct verification of the validity of these results.

Despite the evident similarity between Eqs. (1) and (2), and despite the relevance of Eq. (1) to nonequilibrium work relations, the definition of quantum work given by Eq. (1) might seem ad hoc. Indeed, given the absence of a broadly agreed-upon "textbook" definition of quantum work, one might reasonably suspect that Eq. (1) has been introduced precisely because it leads to quantum nonequilibrium work relations. Furthermore, the wave function collapse that occurs upon measuring the final energy has no classical counterpart. Because of this collapse, the quantum work has no independent physical reality until the measurement is performed at $t=\tau$. By contrast, the classical work can be viewed as a well-defined function of time, namely, the net change in the value of the time-dependent Hamiltonian along the trajectory $z_{t}$. Our aim in this paper is to use the tools of semiclassical mechanics, together with numerical simulations, to investigate the relationship between Eqs. (1) and (2), and specifically to clarify how the correspondence principle applies in this context.

We restrict ourselves to systems with 1 degree of freedom, for which there exist explicit semiclassical approximations of energy eigenstates. We focus on the transition probability $P^{Q}(n \mid m)$ from the $m$ th eigenstate of $\hat{H}_{A}$ to the $n$th eigenstate of $\hat{H}_{B}$, and on its classical analog, $P^{C}(n \mid m)$, defined in Sec. II. In Sec. III, we investigate both quantities numerically for the example of a forced quartic oscillator. For high-lying initial energies $P^{Q}$ oscillates rapidly with $n$, whereas $P^{C}$ is a smooth function over a finite range $n_{\min }<n<n_{\max }$ (Fig. 2). After integrating over the oscillations, the two quantities are nearly identical (Fig. 3), which provides some justification for viewing Eq. (1) as the quantal counterpart of Eq. (2). In Sec. IV, we derive $P^{\mathrm{SC}}(n \mid m)$, a semiclassical approximation for $P^{Q}$ expressed as a sum over classical trajectories. Each trajectory in this sum carries a quantal phase, giving rise to coherent interferences between trajectories. When these interferences are neglected, $P^{\mathrm{SC}}$ agrees with $P^{C}$; when they are included, $P^{\mathrm{SC}}$ accurately captures the oscillations in $P^{Q}$ (Fig. 5). Thus, the oscillations in $P^{Q}$ can be understood as a quantum interference pattern superimposed on a classical background. This picture breaks down around $n_{\min }$ and $n_{\max }$, where $P^{Q}$ exhibits tails that tunnel into classically forbidden regions. In Sec. V, we derive a semiclassical approximation, expressed in terms of the Airy function, that accurately describes these tails (Fig. 9).

The analyses in Secs. IV and V rely on theoretical tools that are familiar in the field of semiclassical mechanics. To the best of our knowledge, these tools have not previously been applied to study the relationship between classical and quantum work distributions, although similar analyses have been performed in the context of molecular scattering theory [65-67] and laser-pulsed atoms [68,69], among other examples. In particular, our calculations in Sec. IV B closely parallel those of Schwieters and Delos [69].

\section{CLASSICAL AND QUANTUM TRANSITION PROBABILITIES}

Here, we introduce notation and specify the problem we plan to study. In Sec. II A, we define a discretized classical work distribution, Eq. (17), that can be compared directly to the quantum work distribution, Eq. (20). These distributions involve classical and quantum transition probabilities, $P^{C}(n \mid m)$ and $P^{Q}(n \mid m)$, which are the central objects of study throughout the rest of the paper.

\section{A. Classical setup}

Consider a system with 1 degree of freedom, described by a Hamiltonian

$$
H(z ; \lambda)=\frac{p^{2}}{2 M}+V(q ; \lambda)
$$

where $z=(q, p)$ denotes a point in phase space and $\lambda$ is an externally controlled parameter. We assume that the energy shells (level surfaces) of the Hamiltonian form simple, closed curves in phase space. We consider the evolution of this system under the time-dependent Hamiltonian $H\left(z ; \lambda_{t}\right)$, where $\lambda$ is varied from $\lambda_{0}=A$ to $\lambda_{\tau}=B$. For compact notation, we define $H_{A}(z) \equiv H\left(z ; \lambda_{0}\right)$ and $H_{B}(z) \equiv H\left(z ; \lambda_{\tau}\right)$.

Suppose that prior to the start of the process the system has come to equilibrium with a thermal reservoir at temperature $T$ and the reservoir has been removed. Therefore, at $t=0$ the microstate of the system will be treated as a random sample from a canonical distribution corresponding to the Hamiltonian $H_{A}$. From $t=0$ to $t=\tau$, the system is described by a trajectory $z_{t}$ evolving under Hamilton's equations, and the work performed on the system is given by the difference between its initial and final energies, as per Eq. (2): 


$$
W=E_{\tau}-E_{0} \equiv H_{B}\left(z_{\tau}\right)-H_{A}\left(z_{0}\right)
$$

For an ensemble of realizations of this process, the distribution of values of work performed on the system is

$P^{C}(W)=\int d E_{\tau} \int d E_{0} \bar{P}^{C}\left(E_{\tau} \mid E_{0}\right) \bar{P}_{A}^{C}\left(E_{0}\right) \delta\left(W-E_{\tau}+E_{0}\right)$,

where $\bar{P}_{A}^{C}\left(E_{0}\right)$ is the probability distribution of initial energies, sampled from equilibrium, and $\bar{P}^{C}\left(E_{\tau} \mid E_{0}\right)$ is the conditional probability distribution to end with a final energy $E_{\tau}$, given an initial energy $E_{0}$. Let us consider these factors separately.

$\bar{P}_{A}^{C}$ is simply the classical equilibrium energy distribution:

$$
\bar{P}_{A}^{C}\left(E_{0}\right)=\frac{1}{Z_{A}^{C}} e^{-\beta E_{0}} g_{A}\left(E_{0}\right),
$$

where

$$
\begin{aligned}
Z_{\lambda}^{C} & =\frac{1}{h} \int d z \exp [-\beta H(z ; \lambda)] \text { and } \\
g_{\lambda}(E) & =\frac{1}{h} \int d z \delta[E-H(z ; \lambda)]
\end{aligned}
$$

are the classical partition function and density of states, $\beta=\left(k_{B} T\right)^{-1}$, and $h$ is Planck's constant. We include the factors $h^{-1}$ [which cancel in Eq. (6)] for later convenience. Let us also define

$$
\Omega(E, \lambda)=\int d z \theta[E-H(z ; \lambda)]=\oint_{E} p d q,
$$

which is the phase space volume enclosed by the energy shell $E$ of the Hamiltonian $H$, equivalently the integral of $p d q$ around this energy shell. We then have

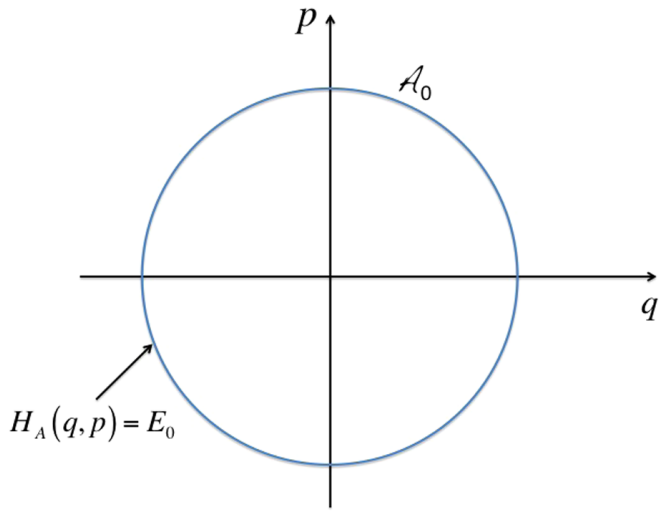

(a)

$$
g_{\lambda}(E)=\frac{1}{h} \frac{\partial \Omega}{\partial E} .
$$

The conditional probability distribution $\bar{P}^{C}\left(E_{\tau} \mid E_{0}\right)$ is given by the expression

$$
\bar{P}^{C}\left(E_{\tau} \mid E_{0}\right)=\frac{\int d z_{0} \delta\left[E_{0}-H_{A}\left(z_{0}\right)\right] \delta\left[E_{\tau}-H_{B}\left(z_{\tau}\left(z_{0}\right)\right)\right]}{\int d z_{0} \delta\left[E_{0}-H_{A}\left(z_{0}\right)\right]},
$$

where $z_{\tau}\left(z_{0}\right)$ denotes the final conditions of a trajectory that evolves from initial conditions $z_{0}$. It is useful to imagine an ensemble of initial conditions sampled microcanonically from the energy shell $E_{0}$ of the Hamiltonian $H_{A}$ [see Fig. 1(a)]. The swarm of trajectories that evolves from these initial conditions defines a time-dependent, closed curve $\mathcal{A}_{t}$ in phase space. At $t=\tau$, the points of intersection between $\mathcal{A}_{\tau}$ and the energy shell $E_{\tau}$ of the Hamiltonian $H_{B}$ [black dots in Fig. 1(b)] represent the final conditions of those trajectories that end with energy $E_{\tau}$, having begun with energy $E_{0}$. If we consider two nearby energy shells $E_{\tau}$ and $E_{\tau}+d E$, then $\bar{P}^{C}\left(E_{\tau} \mid E_{0}\right) d E_{\tau}$ is the fraction of trajectories whose final energies fall within this energy interval.

Let us define the $m$ th energy interval of the Hamiltonian $H_{A}$ to be the range of energy values $E$ satisfying

$$
m h \leq \Omega(E, A)<(m+1) h,
$$

where $m=0,1,2, \ldots$ Furthermore, let us use a midpoint rule to assign a particular energy value $E_{m}^{A}$ to each interval:

$$
\Omega\left(E_{m}^{A}, A\right)=\oint_{E_{m}^{A}} p d q=\left(m+\frac{1}{2}\right) h .
$$

Analogous definitions apply to the $n$th energy interval of the Hamiltonian $H_{B}$ and the corresponding energy $E_{n}^{B}$.

From Eqs. (9) and (11), the width of the $m$ th energy interval of $H_{A}$ is

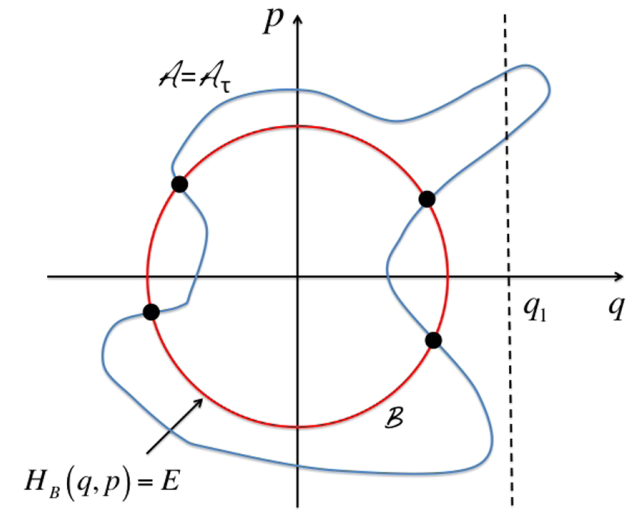

(b)

FIG. 1. An energy shell $\mathcal{A}_{0}$ of the initial Hamiltonian [panel (a), $t=0$ ] evolves under $H(t)$ to the curve $\mathcal{A} \equiv \mathcal{A}_{\tau}$ [panel (b), $t=\tau$ ]. The curve $\mathcal{B}$ is an energy shell of the final Hamiltonian. 


$$
\delta E_{m}^{A} \approx \frac{1}{g_{A}\left(E_{m}^{A}\right)} .
$$

For a smooth function of energy $f(E)$, Eq. (13) leads to

$$
\int_{m} d E_{0} f\left(E_{0}\right) \approx \frac{f\left(E_{m}^{A}\right)}{g_{A}\left(E_{m}^{A}\right)},
$$

where the integral is taken over the $m$ th interval.

Readers will recognize Eq. (12) as the semiclassical quantization condition $\oint p d q=[m+(1 / 2)] h$, which provides an excellent approximation for the $m$ th eigenvalue of the quantum Hamiltonian $\hat{H}_{A}$ [Eq. (18)] when $m \gg 1$. For convenience, we later use the notation $E_{m}^{A}$ to denote this eigenvalue, without making a distinction between the exact eigenvalue and its semiclassical approximation.

The probability to obtain initial conditions $z_{0}$ within the $m$ th interval of $H_{A}$, when sampling from equilibrium, is

$$
P_{A}^{C}(m)=\int_{m} d E_{0} \bar{P}_{A}^{C}\left(E_{0}\right) \approx \frac{1}{Z_{A}^{C}} e^{-\beta E_{m}^{A}}
$$

using Eq. (14). Similarly, the conditional probability to end in the $n$th energy interval, given a representative initial energy in the $m$ th interval, is

$$
P^{C}(n \mid m)=\int_{n} d E_{\tau} \bar{P}^{C}\left(E_{\tau} \mid E_{0}=E_{m}^{A}\right) \approx \frac{\bar{P}^{C}\left(E_{n}^{B} \mid E_{m}^{A}\right)}{g_{B}\left(E_{n}^{B}\right)} .
$$

We refer to this as the classical transition probability. The interpretation of $P^{C}(n \mid m)$ is straightforward. Imagine a swarm of trajectories evolving from initial conditions sampled from a microcanonical ensemble with energy $E_{m}^{A}$ [see Fig. 1]. At the end of the process, $t=\tau$, the fraction of these trajectories that fall into the energy window $\left[E_{n}^{B}, E_{n+1}^{B}\right]$ is equal to $P^{C}(n \mid m)$ [69]. The classical work distribution can now be rewritten as a sum over initial and final energy intervals:

$$
\begin{aligned}
& P^{C}(W) \\
& \quad=\sum_{n, m} \int_{n} d E_{\tau} \int_{m} d E_{0} \bar{P}^{C}\left(E_{\tau} \mid E_{0}\right) \bar{P}_{A}^{C}\left(E_{0}\right) \delta\left(W-E_{\tau}+E_{0}\right) \\
& \quad \approx \sum_{n, m} \int_{n} d E_{\tau} \int_{m} d E_{0} \bar{P}^{C}\left(E_{\tau} \mid E_{m}^{A}\right) \bar{P}_{A}^{C}\left(E_{0}\right) \delta\left(W-E_{n}^{B}+E_{m}^{A}\right) \\
& \quad=\sum_{m, n} P^{C}(n \mid m) P_{A}^{C}(m) \delta\left(W-E_{n}^{B}+E_{m}^{A}\right)
\end{aligned}
$$

Finally, we point out that the approximations appearing in Eqs. (13)-(17) assume that the width of the $m$ th energy interval $\delta E_{m}$ is very small on a classical scale. Formally, this assumption can be justified by taking the semiclassical limit $\hbar \rightarrow 0$ with all classical quantities held fixed. In this limit the density of states increases, as does the quantum number at a given energy. In practice, the semiclassical limit is often simply identified with the condition $m \gg 1$.

\section{B. Quantum setup}

In the quantum version of this problem, $H(z ; \lambda)$ is replaced by the Hermitian operator

$$
\hat{H}(\lambda)=-\frac{\hbar^{2}}{2 M} \frac{\partial^{2}}{\partial q^{2}}+V(q ; \lambda) .
$$

A wave function $\psi(q, t)=\left\langle q \mid \psi_{t}\right\rangle$ evolves under the Schrödinger equation as $\hat{H}\left(\lambda_{t}\right)$ is varied from $\hat{H}\left(\lambda_{0}\right)=$ $\hat{H}_{A}$ to $\hat{H}\left(\lambda_{\tau}\right)=\hat{H}_{B}$. The eigenstates and eigenvalues of $\hat{H}_{A}$ are specified as follows:

$$
\phi_{m}^{A}(q)=\left\langle q \mid \phi_{m}^{A}\right\rangle, \quad \hat{H}_{A}\left|\phi_{m}^{A}\right\rangle=E_{m}^{A}\left|\phi_{m}^{A}\right\rangle,
$$

with similar notation for $\hat{H}_{B}$. Evolution in time is represented by the unitary operator $\hat{U}_{t}$ satisfying $\hat{H}\left(\lambda_{t}\right) \hat{U}_{t}=$ $i \hbar \partial \hat{U}_{t} / \partial t$ and $\hat{U}_{0}=\hat{I}$, where $\hat{I}$ is the identity operator.

Using Eq. (1) and assuming thermal equilibration at $t=0$, the work distribution is [15]

$$
P^{Q}(W)=\sum_{n, m} P^{Q}(n \mid m) P_{A}^{Q}(m) \delta\left(W-E_{n}^{B}+E_{m}^{A}\right) .
$$

$P_{A}^{Q}(m)$ is the probability of obtaining the $m$ th eigenstate of $\hat{H}_{A}$ when making the initial energy measurement:

$$
P_{A}^{Q}(m)=\frac{e^{-\beta E_{m}^{A}}}{Z_{A}^{Q}},
$$

with $Z_{A}^{Q}=\sum_{m} \exp \left(-\beta E_{m}^{A}\right)$. The quantum transition probability $P^{Q}(n \mid m)$ is the conditional probability to obtain the $n$th eigenstate of $\hat{H}_{B}$ upon making the final measurement, given the $m$ th eigenstate of $\hat{H}_{A}$ at the initial measurement:

$$
P^{Q}(n \mid m)=\left|\left\langle\phi_{n}^{B}\left|\hat{U}_{\tau}\right| \phi_{m}^{A}\right\rangle\right|^{2}=\left|\left\langle\phi_{n}^{B} \mid \psi_{\tau}\right\rangle\right|^{2} .
$$

The classical and quantum work distributions given by Eqs. (17) and (20) can now be compared directly. We note that

$$
Z_{A}^{C}=\int d E e^{-\beta E} g_{A}(E) \approx \sum_{m} e^{-\beta E_{m}^{A}}=Z_{A}^{Q}
$$

hence, $P_{A}^{C}(m) \approx P_{A}^{Q}(m)$ [see Eqs. (15) and (21)]. Thus, what remains is to clarify the relationship between the classical and quantum transition probabilities, $P^{C}(n \mid m)$ and $P^{Q}(n \mid m)$. In the following section, we compare these transition probabilities in a model system for which both the classical and quantum dynamics are simulated 


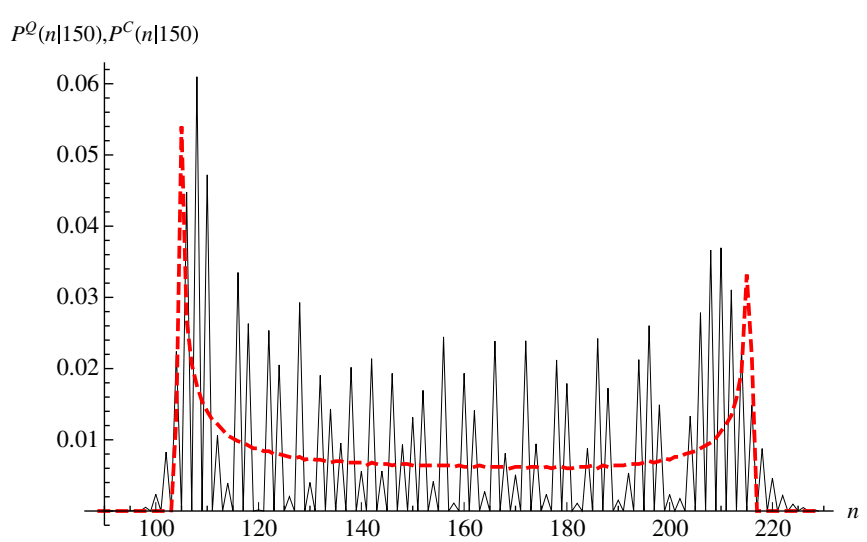

FIG. 2. Quantum [Eq. (22)] and classical [Eq. (16)] transition probabilities for the forced quartic oscillator. The solid black curve shows $P^{Q}(n \mid m)$, while the dashed red curve shows $P^{C}(n \mid m)$, for $m=150$.

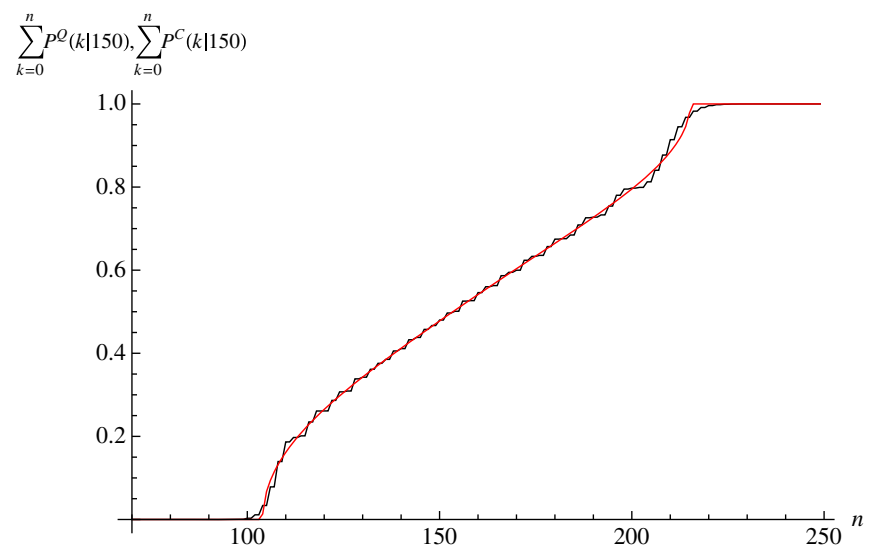

FIG. 3. Accumulated transition probabilities for the forced quartic oscillator. The jagged black curve represents the quantum case, $\sum_{k=0}^{n} P^{Q}(k \mid m)$, while the smooth red curve represents the classical case, $\sum_{k=0}^{n} P^{C}(k \mid m)$.

numerically. We find that $P^{C}$ and $P^{Q}$ are manifestly different (Fig. 2), but these differences mostly vanish after appropriate smoothing (Fig. 3). In Secs. IV and V, we develop a semiclassical theory to explain these features.

\section{NUMERICAL CASE STUDY: THE FORCED QUARTIC OSCILLATOR}

We begin with a model system, the forced quartic oscillator:

$$
H(z ; \lambda)=\frac{p^{2}}{2 M}+\lambda q^{4} .
$$

We set $M=1 / 2$ and $\hbar=h / 2 \pi=1$, and we vary the work parameter at a constant rate, $\lambda_{t}=\lambda_{0}+v t$, from $\lambda_{0}=1=A$ to $\lambda_{\tau}=5=B$, taking $v=50$ and therefore $\tau=0.08$.

A number of groups have previously compared quantum and classical work distributions for a driven harmonic oscillator, for which analytical solutions are available. Specifically, Deffner et al. [70,71] have studied an oscillator with a time-varying stiffness; Talkner et al. [72] have obtained the work distribution for an oscillator driven by time-dependent perturbations that are linear in $q$ and $p$; Campisi [73] has studied changes in the Boltzmann entropy for forced quantum and classical oscillators; Ford et al. [74] have investigated a harmonic oscillator with a cyclically driven stiffness; and Talkner et al. have considered the sudden quench of a two-dimensional oscillator [26]. While the harmonic oscillator has the advantage of being analytically tractable, it is somewhat special in that its quantum dynamics can be reduced to its classical dynamics [75]. Here, we choose the more "generic" quartic oscillator, which requires numerical simulations.

To evaluate $P^{C}(n \mid m)$ we simulate $10^{4}$ Hamiltonian trajectories evolving under $H\left(z ; \lambda_{t}\right)$. Initial conditions are sampled microcanonically, and final conditions are binned according to the energy intervals of $H_{B}(z)$, as defined in Sec. II A. For our initial microcanonical ensemble, we choose $m=150$, corresponding to $E_{0}=E_{m}^{A}=1749.23$. The results are shown by the dashed line in Fig. 2. The transition probability is a smooth function of $n$ from $n_{\min }=105$ to $n_{\max }=215$, and zero outside this range.

These sharp cutoffs reflect the fact that our initial conditions are sampled from a microcanonical distribution: $n_{\min }$ and $n_{\max }$ correspond to the minimal and maximal final energies that can be reached by trajectories launched with initial energies $E_{0}=E_{m}^{A}$, as illustrated in Fig. 7. The characteristic $U$ shape of the classical work distribution within the allowed region is related to the fact that the curves $\mathcal{A}$ and $\mathcal{B}$ shown in Fig. 1(b) become tangent to one another at $E=E_{\min }$ and $E=E_{\max }$ (again, see Fig. 7). For an exactly solvable model in which the same $U$ shape appears, see Fig. 1 of Ref. [73].

For the quantum system, we expand a wave function evolving under $\hat{H}\left(\lambda_{t}\right)=\hat{p}^{2} / 2 M+\lambda_{t} \hat{q}^{4}$ as follows:

$$
\begin{aligned}
\psi(q, t) & =\sum_{n} c_{n}(t)\left\langle q \mid \phi_{n}\left(\lambda_{t}\right)\right\rangle e^{-i \gamma_{n}(t)}, \\
\gamma_{n}(t) & =\frac{1}{\hbar} \int_{0}^{t} E_{n}\left(\lambda_{t^{\prime}}\right) d t^{\prime} .
\end{aligned}
$$

Here, $\hat{H}\left|\phi_{n}\right\rangle=E_{n}\left|\phi_{n}\right\rangle$ and the $c_{n}$ 's are expansion coefficients. The time-dependent Schrödinger equation $i \hbar \dot{\psi}=$ $\hat{H} \psi$ then produces the set of coupled ordinary differential equations,

$$
\begin{aligned}
\dot{c}_{n} & =-\dot{\lambda} \sum_{k}\left\langle\phi_{n} \mid \frac{\partial \phi_{k}}{\partial \lambda}\right\rangle e^{i\left(\gamma_{n}-\gamma_{k}\right)} c_{k} \\
& =-\dot{\lambda} \sum_{k \neq n} \frac{\left\langle\phi_{n}\left|q^{4}\right| \phi_{k}\right\rangle}{E_{k}-E_{n}} e^{i\left(\gamma_{n}-\gamma_{k}\right)} c_{k} .
\end{aligned}
$$


We integrate these equations numerically, from initial conditions $c_{n}(0)=\delta_{m n}$, to obtain

$$
P^{Q}(n \mid m)=\left|c_{n}(\tau)\right|^{2} .
$$

In Fig. 2 we plot the quantum transition probability as a function of the final quantum number $n$ (solid line). Although a correspondence between $P^{Q}(n \mid m)$ and $P^{C}(n \mid m)$ is visually evident, the quantum and classical cases differ in two distinct ways: (i) the quantum probability oscillates rapidly with $n$ and (ii) $P^{Q}(n \mid m)$ shows tails that "tunnel" into the classically forbidden regions $n<n_{\min }$ and $n>n_{\max }$. Both features trace their origin to the wave nature of the quantum system. We also see in Fig. 2 that $P^{Q}(n \mid m)=0$ when $n-m$ is odd. This result follows from the fact that $\left\langle\phi_{n}\left|q^{4}\right| \phi_{k}\right\rangle=0$ when the parities of $n$ and $k$ differ; hence, only transitions between states of the same parity occur under Eq. (26).

In Fig. 3 we plot the accumulated transition probabilities $\sum_{k=0}^{n} P^{Q}(k \mid m)$ and $\sum_{k=0}^{n} P^{C}(k \mid m)$, thereby smoothing out the rapid oscillations in the quantum transition probability. The close agreement observed in Fig. 3 suggests that Eq. (1) is indeed the appropriate quantum counterpart of Eq. (2), though distinctly nonclassical features are visible in the interference and tunneling effects in Fig. 2.

In Secs. IV and V, we investigate these issues analytically. We find that both the agreement seen in Fig. 3 and the quantum effects evident in Fig. 2 can be understood quantitatively, within a semiclassical interpretation in which quantum dynamics are approximated by classical trajectories bearing time-dependent phases.

\section{SEMICLASSICAL THEORY}

In Sec. IVA, we rewrite the classical transition probability $P^{C}(n \mid m)$ [Eq. (16)] as a sum over trajectories that begin and end with energies $E_{m}^{A}$ and $E_{n}^{B}$, respectively [Eq. (39)]. In Sec. IV B we use time-dependent WKB theory to derive a semiclassical approximation $P^{\mathrm{SC}}(n \mid m)$ for the quantum transition probability $P^{Q}(n \mid m)$. Our main result, Eq. (49), is expressed as a sum over the same trajectories that contribute to $P^{C}(n \mid m)$, only now each of these trajectories carries a phase, resulting in interference effects between different trajectories. When these interferences are ignored, we recover the classical transition probability [Eq. (50)]. When they are included, the interferences give rise to the oscillations observed in the quantum transition probability. In particular, interferences between symmetry-related trajectories account for the fastest oscillations observed in Fig. 2, as we discuss in Sec. IV C.

\section{A. Classical transition probabilities}

We begin with the conditional probability distribution $\bar{P}^{C}\left(E \mid E_{0}\right)$ [Eq. (10)], where we drop the subscript $\tau$ from the final energy, for convenience. As discussed in Sec. II, we imagine a swarm of trajectories evolving in time under $H\left(z ; \lambda_{t}\right)$, with initial conditions sampled from the microcanonical phase space distribution,

$$
\eta_{A}\left(z ; E_{0}\right)=\frac{\delta\left[E_{0}-H_{A}(z)\right]}{\int d z \delta\left[E_{0}-H_{A}(z)\right]}=\frac{\delta\left[E_{0}-H_{A}(z)\right]}{h g_{A}\left(E_{0}\right)},
$$

where $E_{0}$ is a parameter of the distribution. The quantity $\bar{P}^{C}\left(E \mid E_{0}\right) d E$ is the fraction of these trajectories that end with a final energy in the infinitesimal range $(E, E+d E)$.

As in Fig. 1, let $\mathcal{A}_{t}$ denote the time-dependent curve that evolves from the initial energy shell $H_{A}=E_{0}$, and let $\mathcal{B}$ denote an energy shell of the final Hamiltonian $H_{B}=E$. The evolving curve $\mathcal{A}_{t}$ can be described by a multivalued, time-dependent momentum field $p_{b}^{\mathcal{A}_{t}}(q)$, where the index $b$ labels the branches of $\mathcal{A}_{t}$ at the coordinate value $q$. For example, in Fig. 1(b), the momentum field for $\mathcal{A}_{\tau}$ has two branches at $q=q_{1}$. Similarly, the multivalued momentum field $p_{b}^{\mathcal{B}}(q)$ describes the fixed energy shell $H_{B}=E$, whose two branches are

$$
p_{b}^{\mathcal{B}}(q)= \pm \sqrt{2 M[E-V(q ; B)]} \quad(b= \pm) .
$$

For convenience, we henceforth use the notation $\mathcal{A}$ (without a subscript) to indicate the surface $\mathcal{A}_{\tau}$. The points of intersection between $\mathcal{A}$ and $\mathcal{B}$, highlighted by dots in Fig. 1(b), represent the trajectories that contribute to $\bar{P}^{C}\left(E \mid E_{0}\right)$.

Our swarm of trajectories at time $t$ is described not only by the evolving surface $\mathcal{A}_{t}$ but also by a probability density on that surface. If we project this density onto the $q$ axis, the projected density $\rho^{\mathcal{A}_{t}}(q)$ is a sum over the branches of the surface $\mathcal{A}_{t}$ :

$$
\rho^{\mathcal{A}_{t}}(q)=\sum_{b} \rho_{b}^{\mathcal{A}_{t}}(q)
$$

The fixed microcanonical ensemble corresponding to $H_{B}=E$ can similarly be projected onto a density

$$
\begin{aligned}
\rho^{\mathcal{B}}(q) & =\frac{1}{h g_{B}(E)} \int d p \delta\left[E-H_{B}(q, p)\right] \\
& =\frac{1}{h g_{B}(E)} \sum_{b}\left|\frac{\partial H_{B}}{\partial p}\right|_{b}^{-1} \equiv \sum_{b} \rho_{b}^{\mathcal{B}}(q),
\end{aligned}
$$

with $\partial H_{B} / \partial p=p / M$ evaluated at $p=p_{b}^{\mathcal{B}}(q)$.

Let $l=1,2, \ldots, K$ be an index labeling the intersection points of $\mathcal{A}$ and $\mathcal{B}$ (e.g., $K=4$ in Fig. 1), and let $b_{l}$ denote the branch corresponding to the $l$ th intersection point:

$$
\left(q_{l}, p_{l}\right)=\left(q_{l}, p_{b_{l}}^{\mathcal{A}}\left(q_{l}\right)\right)=\left(q_{l}, p_{b_{l}}^{\mathcal{B}}\left(q_{l}\right)\right) .
$$


With some abuse of notation, we write $b_{l}$ rather than $b_{l}^{\mathcal{A}}$ and $b_{l}^{\mathcal{B}}$, though in general the $l^{\prime}$ th intersection point may occur at differently indexed branches of the two surfaces.

To evaluate $\bar{P}^{C}\left(E \mid E_{0}\right)$, we begin with the contribution $\bar{P}_{l}^{C}\left(E \mid E_{0}\right)$ from a single intersection point $l$. Figure 4 depicts this intersection, together with an intersection point involving an infinitesimally displaced energy shell $H_{B}=E+\delta E$. The highlighted line segment connecting these two points represents a set of trajectories with final energies between $E$ and $E+\delta E$; hence,

$$
\rho^{\mathcal{A}}\left(q_{l}\right) \cdot|\delta q|=\bar{P}_{l}^{C}\left(E \mid E_{0}\right) \cdot|\delta E| .
$$

Since we focus here on the contribution from a single intersection between $\mathcal{A}$ and $\mathcal{B}$, we suppress the subscripts $b_{l}$ indicating the branches of these surfaces. By construction, the ratio $\delta E / \delta q$ is the rate of change of $H_{B}$ with respect to $q$ along the curve $\mathcal{A}$ :

$$
\frac{\delta E}{\delta q}=\left.\frac{d}{d q} H_{B}\left[q, p^{\mathcal{A}}(q)\right]\right|_{q=q_{l}}
$$

Since $p^{\mathcal{B}}(q)$ specifies a surface of constant $H_{B}$, we have

$$
\frac{\partial p^{\mathcal{B}}}{\partial q}=\left.\frac{\partial p}{\partial q}\right|_{H_{B}}=-\frac{\partial H_{B} / \partial q}{\partial H_{B} / \partial p}
$$

hence,

$$
\begin{aligned}
\frac{d}{d q} H_{B}\left[q, p^{\mathcal{A}}(q)\right] & =\frac{\partial H_{B}}{\partial q}+\frac{\partial H_{B}}{\partial p} \frac{\partial p^{\mathcal{A}}}{\partial q} \\
& =\frac{\partial H_{B}}{\partial p}\left(\frac{\partial p^{\mathcal{A}}}{\partial q}-\frac{\partial p^{\mathcal{B}}}{\partial q}\right) .
\end{aligned}
$$

Combining Eqs. (31), (33), (34), and (36), we get

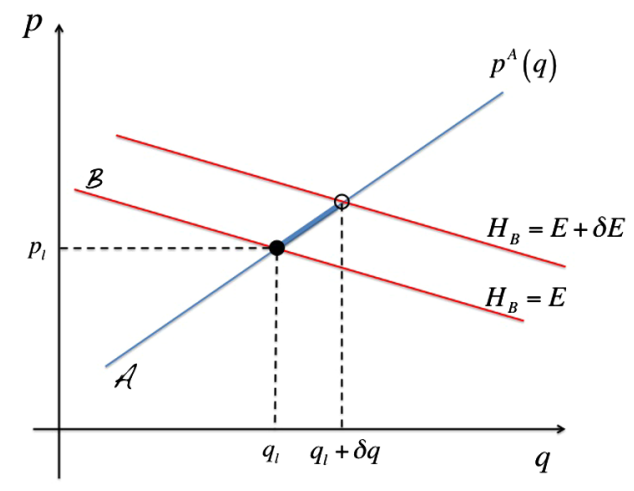

FIG. 4. The filled circle indicates an intersection between the closed curves $\mathcal{A}$ and $\mathcal{B}$; see Fig. 1 . The open circle is an intersection point between $\mathcal{A}$ and a nearby energy shell, $H_{B}=E+\delta E$.

$$
\begin{aligned}
\bar{P}_{l}^{C}\left(E \mid E_{0}\right) & =\rho^{\mathcal{A}}\left(q_{l}\right) \cdot\left|\frac{\delta E}{\delta q}\right|^{-1} \\
& =h g_{B}(E) \rho^{\mathcal{A}}\left(q_{l}\right) \rho^{\mathcal{B}}\left(q_{l}\right)\left|\frac{\partial p^{\mathcal{A}}}{\partial q}-\frac{\partial p^{\mathcal{B}}}{\partial q}\right|_{q=q_{l}}^{-1}
\end{aligned}
$$

Summing over all intersection points gives us

$$
\bar{P}^{C}\left(E \mid E_{0}\right)=h g_{B}(E) \sum_{l=1}^{K} \rho_{b_{l}}^{\mathcal{A}}\left(q_{l}\right) \rho_{b_{l}}^{\mathcal{B}}\left(q_{l}\right)\left|\frac{\partial p_{b_{l}}^{\mathcal{A}}}{\partial q}-\frac{\partial p_{b_{l}}^{\mathcal{B}}}{\partial q}\right|_{q=q_{l}}^{-1}
$$

If we now set $E_{0}=E_{m}^{A}$ and $E=E_{n}^{B}$, then Eq. (16) gives us

$$
P^{C}(n \mid m)=h \sum_{l=1}^{K} \rho_{b_{l}}^{\mathcal{A}}\left(q_{l}\right) \rho_{b_{l}}^{\mathcal{B}}\left(q_{l}\right)\left|\frac{\partial p_{b_{l}}^{\mathcal{A}}}{\partial q}-\frac{\partial p_{b_{l}}^{\mathcal{B}}}{\partial q}\right|_{q=q_{l}}^{-1} .
$$

This is the main result of this subsection.

We use the term classically allowed region to denote the range $E_{\min } \leq E \leq E_{\max }$ within which the function $\bar{P}^{C}\left(E \mid E_{0}\right)$ does not vanish, representing all final energies that can be attained by trajectories with initial energy $E_{0}$. In the coarse-grained function $P^{C}(n \mid m)$, the classically allowed region is bracketed by the values $n_{\min }$ and $n_{\max }$, denoting the energy intervals of $H_{B}$ containing $E_{\min }$ and $E_{\max }$. As illustrated in Fig. 7, the values $E_{\min }$ and $E_{\max }$ correspond, respectively, to the largest energy shell of $H_{B}$ that fits entirely within the closed curve $\mathcal{A}$ and the smallest energy shell of $H_{B}$ that surrounds the entire curve $\mathcal{A}$. At these two energies, the curves $\mathcal{A}$ and $\mathcal{B}$ become tangent to one another, $\partial p_{b_{l}}^{\mathcal{A}} / \partial q=\partial p_{b_{l}}^{\mathcal{B}} / \partial q$, leading to divergences in Eq. (38), which are reflected in the two sharp peaks in $P^{C}(n \mid m)$ in Fig. 2. Similar divergences appear in a more familiar context, namely, the density $\rho^{\mathcal{B}}(q)$ that describes the projection of a fixed microcanonical ensemble $H_{B}=E$ onto the coordinate axis [see Eq. (31)]. In this case, the divergences occur at classical turning points at which the velocity $\partial H_{B} / \partial p$ vanishes.

In the example introduced in Sec. III, the reflection symmetry of the quartic potential implies that trajectories come in symmetry-related pairs: if $\left(q_{t}, p_{t}\right)$ is a solution of Hamilton's equations under the time-dependent Hamiltonian $H\left(q, p ; \lambda_{t}\right)$, then so is $\left(-q_{t},-p_{t}\right)$. This means that for every intersection point $\left(q_{l}, p_{l}\right)$ between $\mathcal{A}$ and $\mathcal{B}$ there will be another intersection point at $\left(-q_{l},-p_{l}\right)$, and the two will contribute equally to $P^{C}(n \mid m)$.

\section{B. Semiclassical transition probabilities}

Let us now evaluate $\bar{P}^{Q}(n \mid m)$ in the semiclassical limit. Appendix A provides a brief introduction to timedependent WKB theory, and for further details we refer the reader to the reviews by Delos [76] and Littlejohn [77]. 
Using Eq. (A5), the wave function $\psi(q, t)=\left\langle q\left|\hat{U}_{t}\right| \phi_{m}^{A}\right\rangle$ and the $n$th eigenstate of $\hat{H}_{B}$ can be written as

$$
\begin{array}{r}
\psi(q, t)=\sum_{b} \sqrt{\rho_{b}^{\mathcal{A}_{t}}(q)} \exp \left[\frac{i}{\hbar} S_{b}^{\mathcal{A}_{t}}(q)-i \mu_{b}^{\mathcal{A}_{t}} \frac{\pi}{2}\right], \\
\phi_{n}^{B}(q)=\sum_{b} \sqrt{\rho_{b}^{\mathcal{B}}(q)} \exp \left[\frac{i}{\hbar} S_{b}^{\mathcal{B}}(q)-i \mu_{b}^{\mathcal{B}} \frac{\pi}{2}\right],
\end{array}
$$

where the actions $S_{b}^{\mathcal{A}_{t}}(q)$ and $S_{b}^{\mathcal{B}}(q)$ generate the momentum fields

$$
p_{b}^{\mathcal{A}_{t}}(q)=\frac{\partial S_{b}^{\mathcal{A}_{t}}}{\partial q}, \quad p_{b}^{\mathcal{B}}(q)=\frac{\partial S_{b}^{\mathcal{B}}}{\partial q},
$$

and the integers $\mu_{b}^{\mathcal{A}_{t}}$ and $\mu_{b}^{\mathcal{B}}$ are Maslov indices (see Appendix A). The densities and momentum fields in Eqs. (40) and (41) are the same as those appearing in Sec. IV A.

From Eq. (40) we get

$$
\begin{aligned}
\left\langle\phi_{n}^{B} \mid \psi_{\tau}\right\rangle= & \sum_{b, b^{\prime}} \int d q \sqrt{\rho_{b}^{\mathcal{A}} \rho_{b^{\prime}}^{\mathcal{B}}} \\
& \times \exp \left[\frac{i}{\hbar}\left(S_{b}^{\mathcal{A}}-S_{b^{\prime}}^{\mathcal{B}}\right)-i\left(\mu_{b}^{\mathcal{A}}-\mu_{b^{\prime}}^{\mathcal{B}}\right) \frac{\pi}{2}\right],
\end{aligned}
$$

which appears as Eq. (37b) in Ref. [69]. Using the stationary phase approximation to evaluate the integral, we obtain a sum of contributions from points $q_{l}$ satisfying the condition

$$
\frac{\partial S_{b}^{\mathcal{A}}}{\partial q}\left(q_{l}\right)=\frac{\partial S_{b^{\prime}}^{\mathcal{B}}}{\partial q}\left(q_{l}\right)
$$

which is equivalent to

$$
p_{b}^{\mathcal{A}}\left(q_{l}\right)=p_{b^{\prime}}^{\mathcal{B}}\left(q_{l}\right) \equiv p_{l}
$$

Hence, $\left\langle\phi_{n}^{B} \mid \psi_{\tau}\right\rangle$ reduces to a sum of contributions arising from the $K$ intersection points of the surfaces $\mathcal{A}$ and $\mathcal{B}$, representing trajectories with initial and final energies $E_{m}^{A}$ and $E_{n}^{B}$, just as in the classical case [Eq. (32)].

To determine the contribution from the $l$ th intersection point, we perform a quadratic expansion around $q=q_{l}$ :

$$
S_{b_{l}}^{\mathcal{A}}(q)-S_{b_{l}}^{\mathcal{B}}(q) \approx \Delta S_{l}+\frac{1}{2} \kappa_{l}\left(q-q_{l}\right)^{2},
$$

with $\Delta S_{l}=S_{b_{l}}^{\mathcal{A}}\left(q_{l}\right)-S_{b_{l}}^{\mathcal{B}}\left(q_{l}\right)$ and

$$
\kappa_{l}=\frac{\partial^{2} S_{b_{l}}^{\mathcal{A}}}{\partial q^{2}}\left(q_{l}\right)-\frac{\partial^{2} S_{b_{l}}^{\mathcal{B}}}{\partial q^{2}}\left(q_{l}\right)=\frac{\partial p_{b_{l}}^{\mathcal{A}}}{\partial q}\left(q_{l}\right)-\frac{\partial p_{b_{l}}^{\mathcal{B}}}{\partial q}\left(q_{l}\right) .
$$

Performing the stationary phase integral then gives us

$$
\begin{aligned}
\left\langle\phi_{n}^{B} \mid \psi_{\tau}\right\rangle \approx & \sum_{l=1}^{K} \sqrt{\rho_{b_{l}}^{\mathcal{A}} \rho_{b_{l}}^{\mathcal{B}}} \exp \left(\frac{i}{\hbar} \Delta S_{l}-i \Delta \mu_{l} \frac{\pi}{2}\right) \\
& \times \int d q \exp \left[\frac{i \kappa_{l}}{2 \hbar}\left(q-q_{l}\right)^{2}\right] \\
= & \sum_{l=1}^{K} \sqrt{\rho_{b_{l}}^{\mathcal{A}} \rho_{b_{l}}^{\mathcal{B}}} \exp \left(\frac{i}{\hbar} \Delta S_{l}-i \Delta \mu_{l} \frac{\pi}{2}\right) \sqrt{\frac{2 \pi \hbar}{\left|\kappa_{l}\right|}} e^{i \sigma_{l} \pi / 4} \\
= & \sum_{l} a_{l} e^{i \theta_{l}},
\end{aligned}
$$

where $\rho_{b_{l}}^{\mathcal{A}, \mathcal{B}}$ are evaluated at $q=q_{l} ; \Delta \mu_{l}=\mu_{b_{l}}^{\mathcal{A}}-\mu_{b_{l}}^{\mathcal{B}}$; and $\sigma_{l}=\operatorname{sgn}\left(\kappa_{l}\right)= \pm 1$. In Ref. [69], where similar calculations are performed, a quantity equivalent to our $\pi \sigma_{l} / 4$ is identified as a Maslov-like phase (Ref. [69], p. 1037).

Equation (47) expresses $\left\langle\phi_{n}^{B} \mid \psi_{\tau}\right\rangle$ as a sum over trajectories, each contributing an amplitude and a phase:

$$
a_{l}=\sqrt{2 \pi \hbar \frac{\rho_{b_{l}}^{\mathcal{A}} \rho_{b_{l}}^{\mathcal{B}}}{\left|\kappa_{l}\right|}}, \quad \theta_{l}=\frac{1}{\hbar} \Delta S_{l}-\frac{\pi}{2} \Delta \mu_{l}+\frac{\pi}{4} \sigma_{l} .
$$

The semiclassical transition probability $P^{\mathrm{SC}}(n \mid m) \approx$ $\left|\left\langle\phi_{n}^{B} \mid \psi_{\tau}\right\rangle\right|^{2}$ is then given by

$$
P^{\mathrm{SC}}(n \mid m)=\left|\sum_{l=1}^{K} a_{l} e^{i \theta_{l}}\right|^{2} .
$$

The general form of Eq. (49) is not surprising. The path integral formulation of quantum mechanics tells us that solutions of the time-dependent Schrödinger equation can be represented in terms of sums over classical paths decorated by phases $\exp (i S / \hbar)$, and when $\hbar \rightarrow 0$ the dominant contributions to the sums come from those paths that are solutions of the classical equations of motion [78].

We expect the semiclassical transition probability function $P^{\mathrm{SC}}(n \mid m)$ to provide a good approximation to the quantum transition probability $P^{Q}(n \mid m)$, for large quantum numbers. For the forced quartic oscillator, Fig. 5 compares these functions, taking $m=150 . P^{Q}$ is computed as described in Sec. III, and $P^{\mathrm{SC}}$ is evaluated directly from classical simulations of trajectories evolving from an initial microcanonical ensemble. (The evolution of the multivalued action $S^{\mathcal{A}_{t}}$ is obtained by integrating $p d q-H d t$ along each trajectory; see, e.g., Sec. 3.5 of Ref. [77].) We observe that the agreement is excellent, except in the vicinity of the boundaries between the classically allowed 


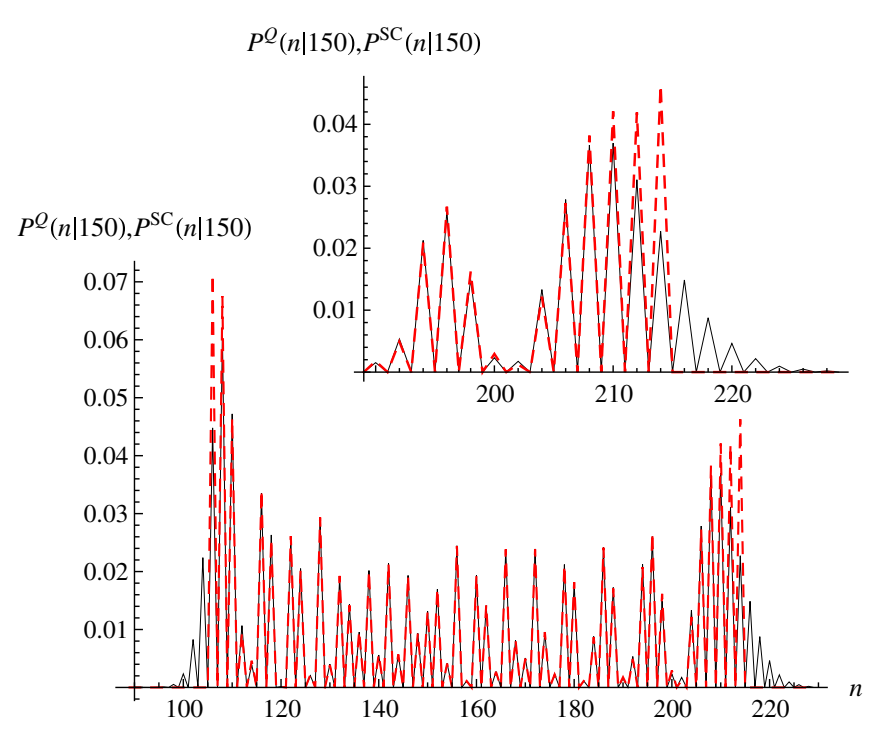

FIG. 5. A comparison of quantum and semiclassical transition probabilities for our model system. The black solid curve shows $P^{Q}(n \mid m)$, while the red dashed curve shows $P^{\mathrm{SC}}(n \mid m)$, as given by Eq. (49). All of the parameters are the same as those in Fig. 2.

and forbidden regions. We examine these boundaries in more detail in Sec. V.

Now let us simplify Eq. (49) by simply ignoring the cross terms in the double sum. This is the so-called diagonal approximation [79-81], and it leads to

$$
P^{\mathrm{SC}}(n \mid m) \stackrel{\operatorname{diag}}{\approx} \sum_{l=1}^{K} a_{l}^{2}=2 \pi \hbar \sum_{l=1}^{K} \rho_{b_{l}}^{\mathcal{A}} \rho_{b_{l}}^{\mathcal{B}}\left|\frac{\partial p_{b_{l}}^{\mathcal{A}}}{\partial q}-\frac{\partial p_{b_{l}}^{\mathcal{B}}}{\partial q}\right|_{q=q_{l}}^{-1}
$$

which is identical to the expression for the classical transition probability, $P^{C}(n \mid m)$ [Eq. (39)].

The agreement between $P^{Q}$ and $P^{\mathrm{SC}}=\left|\sum a_{l} e^{i \theta_{l}}\right|^{2}$ seen in Fig. 5 suggests that the rapid oscillations of the quantum probability distribution can be understood in terms of phase interference between different trajectories. When these interferences are ignored, we recover the classical probability distribution, as we see in Eq. (50). Hence, $P^{\mathrm{SC}}$ acts as a bridge between the quantum and classical transition probabilities,

$$
P^{Q}(n \mid m) \approx P^{\mathrm{SC}}(n \mid m) \stackrel{\text { diag }}{\approx} P^{C}(n \mid m)
$$

and thus between the quantum and classical work distributions [Eqs. (20) and (17)]. The first approximation in Eq. (51) involves time-dependent WKB theory together with a stationary phase evaluation of integrals, and the second is the diagonal approximation in which interferences are ignored.

\section{Interferences and symmetries}

Let us now examine the interferences in more detail. Recall from Sec. IVA that the trajectories contributing to Eq. (49) come in symmetry-related pairs. This allows us to write

$$
\left\langle\phi_{n}^{B} \mid \psi_{\tau}\right\rangle=\sum_{l=1}^{K} a_{l} e^{i \theta_{l}}=\sum_{l=1}^{J}\left(a_{l} e^{i \theta_{l}}+a_{l+J} e^{i \theta_{l+J}}\right),
$$

where $J=K / 2$ is an integer, and the $l$ th and $(l+J)$ th trajectories are related by symmetry:

$$
\left(q_{l+J}, p_{l+J}\right)=\left(-q_{l},-p_{l}\right) .
$$

Here, we index the points $\left(q_{l}, p_{l}\right)$ from $l=1$ to $K$, according to the order in which they appear as we proceed clockwise around the energy shell $\mathcal{B}$, first rightward along the upper branch and then leftward along the lower branch. Equation (53) implies

$$
a_{l+J}=a_{l}, \quad \sigma_{l+J}=\sigma_{l} .
$$

Using the fact that the points $l$ and $l+J$ are located directly opposite one another on the closed curves $\mathcal{A}$ and $\mathcal{B}$ [Eq. (53)], we obtain

$$
\begin{aligned}
& S_{l+J}^{\mathcal{A}}-S_{l}^{\mathcal{A}}=\frac{1}{2} \oint_{\mathcal{A}} p d q=\left(m+\frac{1}{2}\right) \pi \hbar, \\
& S_{l+J}^{\mathcal{B}}-S_{l}^{\mathcal{B}}=\frac{1}{2} \oint_{\mathcal{B}} p d q=\left(n+\frac{1}{2}\right) \pi \hbar,
\end{aligned}
$$

and

$$
\mu_{l+J}^{\mathcal{A}}-\mu_{l}^{\mathcal{A}}=\mu_{l+J}^{\mathcal{B}}-\mu_{l}^{\mathcal{B}}=1
$$

hence,

$$
\Delta S_{l+J}=\Delta S_{l}+(m-n) \pi \hbar, \quad \Delta \mu_{l+J}=\Delta \mu_{l} .
$$

[In Eq. (55) we invoke the semiclassical quantization condition, Eq. (12), together with Liouville's theorem, which guarantees that $\oint_{\mathcal{A}_{t}} p d q$ remains constant with time. In Eq. (56) we use the fact that the Maslov index is incremented by +2 as one proceeds around the closed curve $\mathcal{A}$ or $\mathcal{B}$; see, e.g., Fig. 1 of Ref. [69].] Equations (48), (52), (54), and (57) give us

$$
\left\langle\phi_{n}^{B} \mid \psi_{\tau}\right\rangle=\sum_{l=1}^{J} a_{l} e^{i \theta_{l}}\left[1+e^{i(m-n) \pi}\right] .
$$

When $m-n$ is even, the symmetry-related trajectories interfere constructively, but when $m$ and $n$ have opposite parities, they interfere destructively and we get $P^{\mathrm{SC}}(n \mid m)=0$. This provides a semiclassical explanation 
for the observation, noted in Sec. III, that the quantum transition probability vanishes when $m-n$ is odd: the most rapid oscillations in $P^{Q}(n \mid m)$ arise from interference between symmetry-related trajectories. The same interpretation was given by Miller to explain angular momentum selection rules in the scattering matrix describing an atom impinging on a homonuclear diatomic molecule (see Sec. III C of Ref. [67]).

The interference effects in $P^{\mathrm{SC}}$, due to cross terms in Eq. (49), include contributions not only from pairs of trajectories that are related by symmetry [Eq. (53)] but also from those that are not related by symmetry:

$$
P^{\mathrm{SC}}(n \mid m)=\sum_{l=1}^{K} a_{l}^{2}+\sum_{l \neq k}^{s} a_{l} a_{k} e^{i\left(\theta_{l}-\theta_{k}\right)}+\sum_{l \neq k}^{n} a_{l} a_{k} e^{i\left(\theta_{l}-\theta_{k}\right)},
$$

where $\sum^{s}$ denotes a sum of symmetry-related pairs of trajectories, $\left(q_{k}, p_{k}\right)=-\left(q_{l}, p_{l}\right)$, and $\sum^{n}$ is a sum over nonsymmetry-related pairs, $\left(q_{k}, p_{k}\right) \neq-\left(q_{l}, p_{l}\right)$. To illustrate these contributions separately, we construct the quantity $P_{\text {sym }}^{\mathrm{SC}}$ by omitting the sum $\sum^{n}$ on the right-hand side of Eq. (59), and we similarly construct $P_{\text {nosym }}^{\text {SC }}$ by omitting $\sum^{s}$. The results are shown in Fig. 6. $P_{\mathrm{sym}}^{\mathrm{SC}}$ displays a regular oscillation, as the symmetry-related trajectories are either exactly in phase or exactly out of phase, according to the parity of $m-n$. The oscillations in $P_{\text {nosym }}^{\mathrm{SC}}$ are less regular, as the phases $\theta_{l}-\theta_{k}$ are not necessarily integer multiples of $\pi$. When all cross terms are included, both sets of oscillations combine to give the pattern in Fig. 5. For instance, the gap observed near $n=200$ in Fig. 5 reflects the corresponding minimum in $P_{\text {nosym }}^{\text {SC }}$ seen in Fig. 6(b).

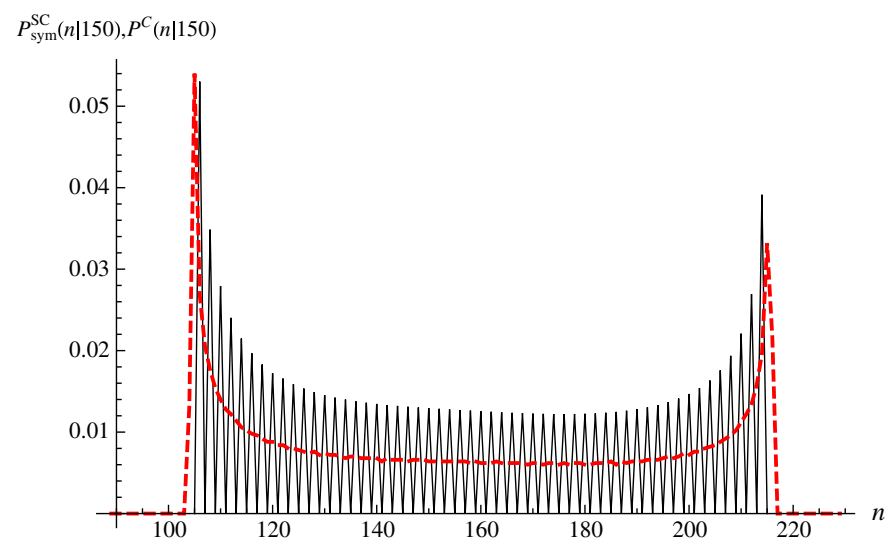

(a)

\section{AIRY TAILS}

In Fig. 5 we see that our semiclassical approximation fails around the boundaries of the classical allowed region at $n_{\min }$ and $n_{\max }$. Although the semiclassical transition probability derived in Sec. IV vanishes identically outside the classically allowed region (since only classical trajectories contribute to $P^{\mathrm{SC}}$ ), the quantum transition probability tunnels into the classically forbidden region, as seen in Fig. 5. In this section, we construct a semiclassical approximation that describes this behavior. Our central results are given by Eqs. (64) and (69) and illustrated in Fig. 9.

Similar tunneling behavior is observed in the wave functions of energy eigenstates, which exhibit tails that reach into classically forbidden regions [82], as well as in semiclassical treatments of the $S$ matrix in molecular collisions [66]; in both cases the tails are approximated by Airy functions. The expressions that we obtain in this section are also expressed in terms of Airy functions.

The failure of $P^{\mathrm{SC}}(n \mid m)$ in the boundary regions originates in the fact that the curves $\mathcal{A}$ and $\mathcal{B}$ become tangent to one another at $E=E_{\min }$ and $E=E_{\max }$. In the example we study numerically, the curves are tangent simultaneously at two points, due to the symmetry of the quartic potential, as shown in Fig. 7(b). However, it is useful first to discuss the generic case, where there exists only a single point of tangency, depicted in Fig. 7(a). For specificity, we discuss the upper boundary of the classically allowed region at $E_{\max }$, but similar comments apply to the lower boundary at $E_{\min }$.

Let us consider the function

$$
\Delta S(q, E)=S^{\mathcal{A}}(q)-S^{\mathcal{B}}(q, E)
$$

that appears inside the exponent in Eq. (42) and its derivative

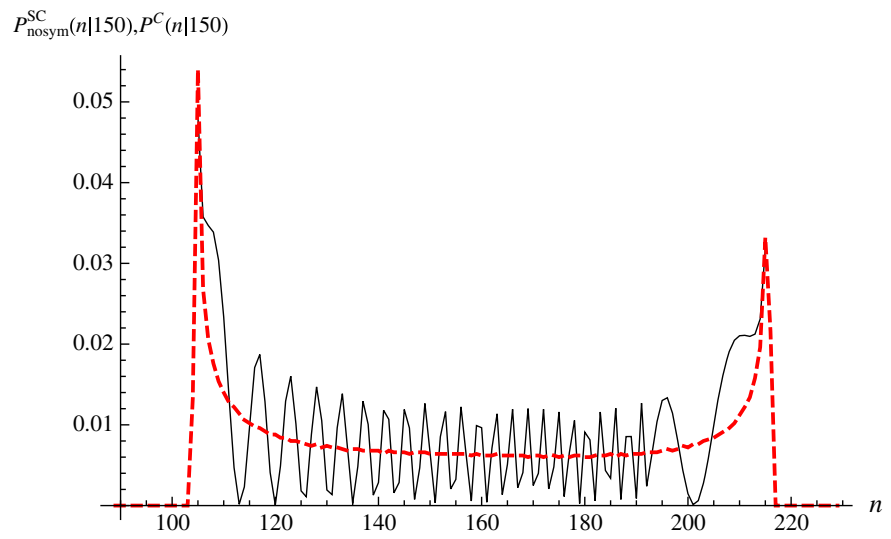

(b)

FIG. 6. Contributions to the interference pattern in $P^{\mathrm{SC}}(n \mid m)$. Panel (a) includes only interferences between symmetry-related trajectories, while (b) includes only interferences between nonsymmetry-related trajectories. 


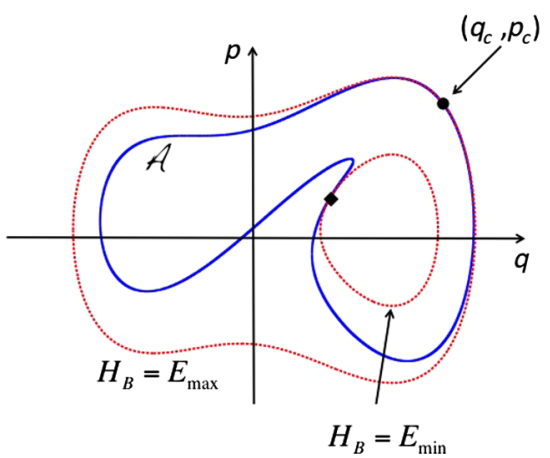

(a)

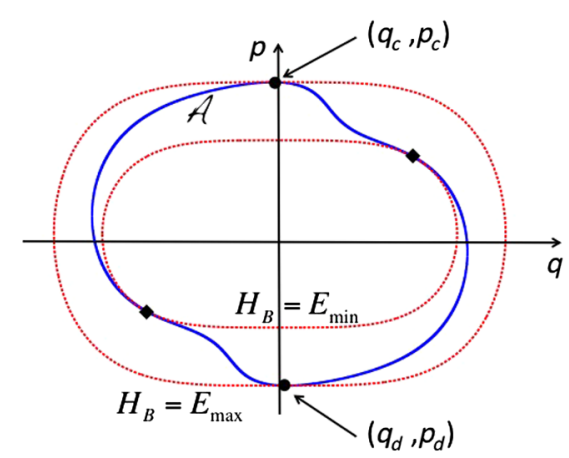

(b)

FIG. 7. Solid blue lines depict the surfaces $\mathcal{A}=\mathcal{A}_{\tau}$ (as in Fig. 1). Dashed red lines are energy shells $\mathcal{B}$ of $H_{B}$. Diamonds and dots indicate the points of tangency between $\mathcal{A}$ and $\mathcal{B}$ at $E_{\min }$ and $E_{\max }$, respectively. (a) In the absence of symmetries, we generically expect a single tangent point at $E_{\min }$ and $E_{\max }$. (b) For the Hamiltonian that we study numerically, Eq. (24), the symmetry of the quartic potential implies that both $\mathcal{A}$ and the energy shells $\mathcal{B}$ are symmetric under $(q, p) \rightarrow(-q,-p)$; thus, points of tangency come in symmetry-related pairs.

$$
\Delta p(q, E)=p^{\mathcal{A}}(q)-p^{\mathcal{B}}(q, E)=\frac{\partial}{\partial q} \Delta S(q, E)
$$

We suppress the subscripts $b$ and $b^{\prime}$, and we explicitly indicate that $S^{\mathcal{B}}$ and $p^{\mathcal{B}}$ depend on the final energy shell $E$. In Sec. IV, we evaluate the integral $\int d q \exp (i \Delta S / \hbar)$ by performing a quadratic expansion of $\Delta S$ around a single intersection point $q_{l}$ between the curves $\mathcal{A}$ and $\mathcal{B}$, where $\Delta p$ vanishes (Fig. 4 ). As the energy $E$ approaches $E_{\max }$ from below, two points of intersection between $\mathcal{A}$ and $\mathcal{B}$ coalesce at a point $\left(q_{c}, p_{c}\right)$, indicated in Fig. 7(a). Figure 8 illustrates the behavior of $\Delta p(q, E)$ and $\Delta S(q, E)$ in the vicinity of $q_{c}$ and $E_{\max }$. In particular, $\Delta S$ has two stationary points when $E<E_{\max }$ and none when $E>E_{\max }$. The stationary phase integration we perform in Sec. IV [Eqs. (43)-(47)] implicitly assumes well-isolated stationary points, but this assumption breaks down near $E=E_{\max }$. In this situation, we evaluate the integral $\int d q \exp (i \Delta S / \hbar)$ by expanding $\Delta S$ to cubic rather than quadratic order. Leaving the details of the calculation to Appendix B, here we present the result:

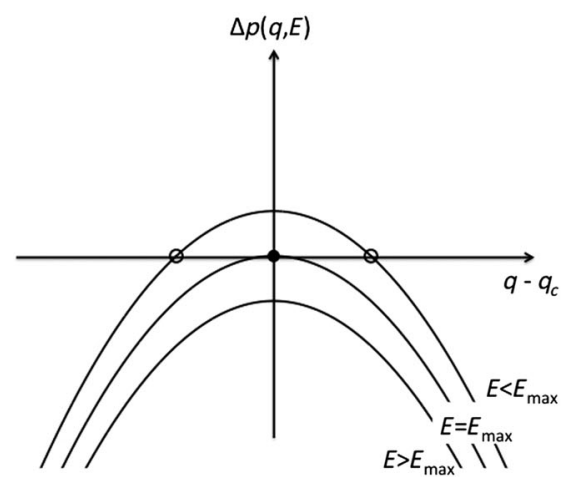

$$
\begin{aligned}
& \int d q \exp \left[\frac{i}{\hbar} \Delta S(q, E)\right] \\
& =2 \pi\left(\frac{2 \hbar}{|k|}\right)^{1 / 3} \exp \left[\frac{i}{\hbar} \Delta S\left(q_{c}, E\right)\right] \\
& \times \mathrm{Ai}\left[-\frac{2^{1 / 3} \nu}{k^{1 / 3} \hbar^{2 / 3}}\left(E-E_{\max }\right)\right]
\end{aligned}
$$

where

$$
k=\frac{\partial^{2} \Delta p}{\partial q^{2}}\left(q_{c}, E_{\max }\right), \quad \nu=\frac{\partial p^{\mathcal{B}}}{\partial E}\left(q_{c}, E_{\max }\right)
$$

and $\operatorname{Ai}(\cdot)$ denotes the Airy function. Combining this result with Eq. (42), we obtain the following expression, which is valid when $E_{n}^{B} \approx E_{\max }$ :

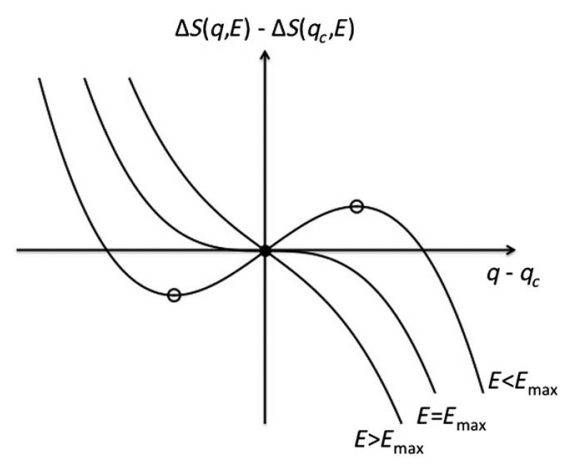

FIG. 8. Behavior of $\Delta p$ and $\Delta S$ for $E \approx E_{\max }$. The open circles correspond to intersection points between $\mathcal{A}$ and $\mathcal{B}$ when $E<E_{\max }$. These coalesce into a single point of tangency at $E=E_{\max }$ (solid circle), and for $E>E_{\max }, \mathcal{A}$ and $\mathcal{B}$ do not intersect. 


$$
\begin{aligned}
& P_{1}^{\mathrm{SC}, \text { tail }}(n \mid m) \\
& \quad=\left|\left\langle\phi_{n}^{B} \mid \psi_{\tau}\right\rangle\right|^{2} \\
& \quad=4 \pi^{2} \rho_{c}^{\mathcal{A}} \rho_{c}^{\mathcal{B}}\left(\frac{2 \hbar}{|k|}\right)^{2 / 3}\left|\mathrm{Ai}\left[-\frac{2^{1 / 3} \nu}{k^{1 / 3} \hbar^{2 / 3}}\left(E_{n}^{B}-E_{\max }\right)\right]\right|^{2},
\end{aligned}
$$

where $\rho_{c}^{\mathcal{A}, B}=\rho^{\mathcal{A}, B}\left(q_{c}\right)$, and the subscript 1 emphasizes that we assume only a single point of tangency between $\mathcal{A}$ and $\mathcal{B}$. At the lower boundary of the classically allowed region, we obtain the same result but with $E_{\max }$ replaced by $E_{\min }$ in Eqs. (63) and (64).

The Airy function decays rapidly for positive values of its argument, and oscillates for negative values:

$$
\begin{aligned}
\operatorname{Ai}(\zeta) & \sim \frac{1}{2 \sqrt{\pi}} \zeta^{-1 / 4} \exp \left(-\frac{2}{3} \zeta^{3 / 2}\right), \\
\operatorname{Ai}(-\zeta) & \sim \frac{1}{\sqrt{\pi}} \zeta^{-1 / 4} \sin \left(\frac{2}{3} \zeta^{3 / 2}+\frac{\pi}{4}\right),
\end{aligned}
$$

for real $\zeta \gg 1$. One can establish by inspection that $\nu>0>k$ when $E>E_{\max }$ and $\nu, k>0$ when $E<E_{\min }$; therefore, the argument of the Airy function in $P_{1}^{\mathrm{SC} \text {,tail }}$ is positive outside the classically allowed region. As a result, the transition probability decays monotonically, penetrating the classically forbidden region over a characteristic skin depth $\left|k^{1 / 3} \hbar^{2 / 3} \nu^{-1}\right|$.

Let us now consider the situation corresponding to the example of Sec. III, in which there are simultaneously two points of tangency between the curves $\mathcal{A}$ and $\mathcal{B}$ at $E=E_{\max }$, and these two points are related by symmetry [Fig. 7(b)]. Using the subscripts $c$ and $d$ to distinguish these two points, we have

$$
\begin{aligned}
\left(q_{c}, p_{c}\right) & =\left(-q_{d},-p_{d}\right), \quad \nu_{c}=-\nu_{d} \equiv \nu, \\
k_{c} & =-k_{d} \equiv k, \quad \rho_{c}^{\mathcal{A}, \mathcal{B}}=\rho_{d}^{\mathcal{A}, \mathcal{B}} .
\end{aligned}
$$

Summing the contributions from these two points gives us

$$
\begin{aligned}
\left\langle\phi_{n}^{B} \mid \psi_{\tau}\right\rangle= & 2 \pi \sqrt{\rho_{c}^{\mathcal{A}} \rho_{c}^{\mathcal{B}}}\left(\frac{2 \hbar}{|k|}\right)^{1 / 3} \mathrm{Ai}\left[-\frac{2^{1 / 3} \nu}{k^{1 / 3} \hbar^{2 / 3}}\left(E-E_{\max }\right)\right] \\
& \times\left[e^{i\left(\Delta S_{c} / \hbar-\Delta \mu_{c} \pi / 2\right)}+e^{i\left(\Delta S_{d} / \hbar-\Delta \mu_{d} \pi / 2\right)}\right],
\end{aligned}
$$

where $\Delta S_{c, d}$ are evaluated at $E=E_{\max }$. Following arguments similar to those leading to Eq. (58), we obtain

$$
\begin{aligned}
& e^{i\left(\Delta S_{c} / \hbar-\Delta \mu_{c} \pi / 2\right)}+e^{i\left(\Delta S_{d} / \hbar-\Delta \mu_{d} \pi / 2\right)} \\
& =e^{i\left(\Delta S_{c} / \hbar-\Delta \mu_{c} \pi / 2\right)}\left[1+e^{i(m-n) \pi}\right] .
\end{aligned}
$$

Equations (67) and (68) lead to a result identical to Eq. (64), apart from a factor that captures the interference between the two parity-related trajectories:

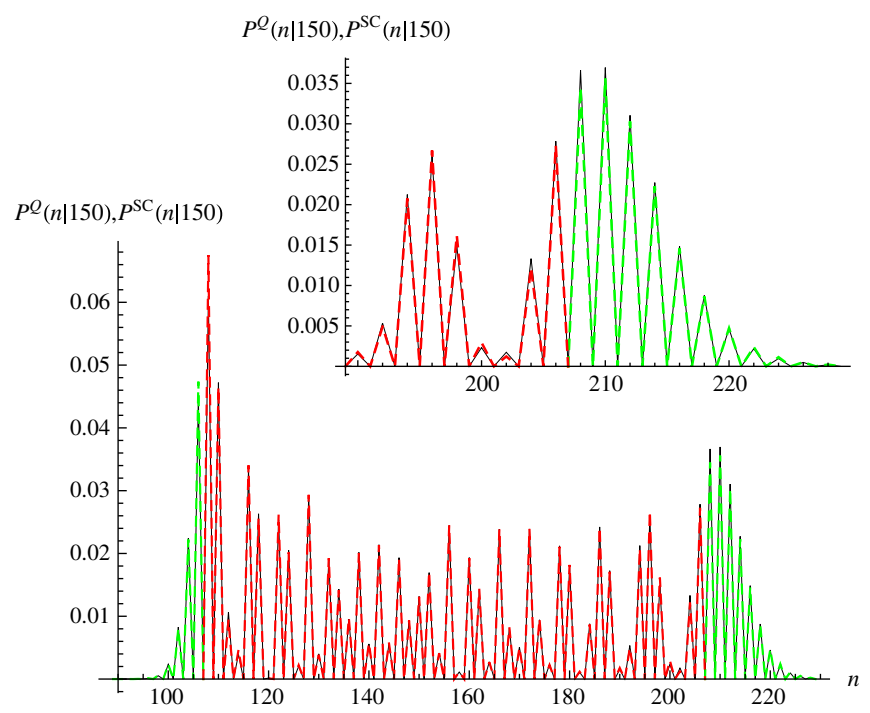

FIG. 9. Comparison between the quantum transition probabilities evaluated by numerical integration of the Schrödinger equation (black, solid line) and the semiclassical approximations given by Eq. (49) in the central region $110<n<200$ (red, dashed line) and by Eq. (69) in the boundary regions $90<n<$ 110 and $200<n<230$ (green, dashed line).

$$
P_{2}^{\mathrm{SC}, \text { tail }}(n \mid m)=P_{1}^{\mathrm{SC}, \text { tail }}(n \mid m) \cdot 4 \cos ^{2}\left[\frac{(m-n) \pi}{2}\right],
$$

with the subscript indicating two points of tangency between $\mathcal{A}$ and $\mathcal{B}$.

Figure 9 compares the quantum transition probability $P^{Q}$ with our semiclassical approximations given by $P^{\mathrm{SC}}$ [Eq. (49)] and $P_{2}^{\mathrm{SC} \text {,tail }}$ [Eq. (69)], in the central and boundary regions, respectively. The agreement is excellent, illustrating that the entire quantum work distribution can be understood in terms of contributions from individual classical trajectories and the interferences between them.

\section{DISCUSSION AND CONCLUSION}

The correspondence principle broadly states that classical mechanics is recovered from quantum mechanics in the limit of large quantum numbers. In textbook discussions, this principle is often illustrated in terms of static properties, typically by comparing the coordinate space probability distribution of a harmonic oscillator eigenstate with the classical, microcanonical distribution at the same energy $[82,83]$. Here, by contrast, we consider the correspondence principle in a dynamic setting, comparing classical and quantum transition probabilities under a time-dependent Hamiltonian. In this setting, timedependent WKB theory provides a set of tools for using classical trajectories to construct approximate solutions of quantum dynamics.

We apply these tools to study the relationship between the definitions of quantum and classical work given by Eqs. (1) and (2). Focusing on the transition probabilities 
$P^{Q}(n \mid m)$ and $P^{C}(n \mid m)$ for systems with 1 degree of freedom, we obtain three main conclusions, and illustrate them with simulations of a driven quartic oscillator:

(i) In the classically allowed region, the quantum transition probability can be approximated in terms of interfering classical trajectories [Eq. (49), Fig. 5].

(ii) When the interferences between these trajectories are ignored, the classical transition probability is obtained [Eq. (50), Fig. 3].

(iii) The tunneling of the quantum transition probability into the classically forbidden region is accurately described by a decaying Airy function, whose arguments are expressed in terms of classical quantities [Eqs. (64) and (69), Fig. 9].

The second conclusion, and Fig. 3 in particular, clarifies the sense in which classical mechanics is "recovered" in the limit of large quantum numbers (in this context), whereas the first and third conclusions describe inherently quantal effects-interference, tunneling - that can nevertheless be understood and approximated in terms of classical trajectories. In view of similar analyses in atomic and molecular contexts (see, e.g., Refs. [65-69]), these conclusions are not surprising. However, given recent interest in quantum nonequilibrium work relations, our results provide a timely investigation of the correspondence principle as it applies to the work performed on a system driven away from equilibrium. As we show, semiclassical mechanics provides a bridge between classical and quantal work distributions, and our conclusions provide some justification for the twopoint measurement definition of quantum work [Eq. (1)].

It will be interesting to study whether our results generalize to systems with more than 1 degree of freedom. For $N$-particle systems with $N \gg 1$, we generically expect that the classical transition probability $P^{C}(n \mid m)$ will be bell shaped rather than $\mathrm{U}$ shaped, with $n_{\min }$ and $n_{\max }$ found deep in the tails of $P^{C}(n \mid m)$. In this situation the quantum tunneling into the forbidden regions will be negligible, since both $P^{C}(n \mid m)$ and $P^{Q}(n \mid m)$ will be very small at $n_{\text {min }}$ and $n_{\max }$. An obstacle to studying $N$ particle systems analytically is the lack of semiclassical representations of energy eigenstates, analogous to Eq. (40b), for generic systems with multiple degrees of freedom. Progress might be made by studying two limiting cases: integrable and fully chaotic systems [84]. For systems whose classical dynamics are integrable, semiclassical expressions for energy eigenstates can be constructed using classical action-angle variables [see, e.g., Eq. (11) of Ref. [85], Eq. (3.5) of Ref. [86], or Eq. (37) of Ref. [87]]. At the other extreme, for fully chaotic systems, Berry's conjecture [88] suggests that energy eigenstates can be treated as Gaussian random functions of the configuration $\mathbf{q}$. In either case, the expression for the energy eigenstate might be combined with time-dependent WKB theory as a first step toward generalizing Eq. (42) and subsequent results to multidimensional systems.

\section{ACKNOWLEDGMENTS}

H. T. Q. acknowledges Meng-Li Du for helpful discussions. C. J. gratefully acknowledges support from the National Science Foundation (U.S.) under Grants No. DMR-1206971 and No. DMR-1506969. H. T. Q. gratefully acknowledges support from the National Science Foundation of China under Grant No. 11375012, and The Recruitment Program of Global Youth Experts of China. S. R. and C. J. are grateful for support from the U.S.-Israel Binational Science Foundation (Grant No. 2010363). S. R. gratefully acknowledges the support of the Israel Science Foundation (Grant No. 924/ 11 ). This work was partially supported by the COST Action MP1209.

\section{APPENDIX A: BRIEF REVIEW OF TIME-DEPENDENT WKB THEORY}

In time-dependent WKB theory, approximate solutions of the time-dependent Schrödinger equation are expressed in terms of classical constructions in phase space. In this brief summary, we restrict ourselves to a single degree of freedom and a Hamiltonian of the form $H(q, p, t)=$ $p^{2} / 2 M+V(q, t)$.

A wave function $\psi(q, t)$ is said to be in WKB form when written as the product of a slowly varying amplitude and a rapidly oscillating phase,

$$
\psi(q, t)=A(q, t) \exp \left[\frac{i}{\hbar} S(q, t)\right],
$$

or else as a sum of such terms, as in Eq. (A5). Locally, Eq. (A1) describes a wave train $\psi \propto \exp (i k q)$, with wave number $k=\hbar^{-1} \partial S / \partial q$. This implies a local momentum

$$
p(q, t)=\hbar k=\frac{\partial S}{\partial q}(q, t),
$$

and the Born rule implies a probability density

$$
\rho(q, t)=|A(q, t)|^{2} .
$$

These functions inherit their time dependence from $\psi(q, t)$. A standard calculation, in which Eq. (A1) is substituted into the Schrödinger equation and $\hbar$ is treated as a small parameter [77] produces the equations of motion

$$
\begin{gathered}
\frac{\partial S}{\partial t}+H\left(q, \frac{\partial S}{\partial q}, t\right)=0, \\
\frac{\partial \rho}{\partial t}+\frac{\partial}{\partial q}\left(\frac{p}{M} \rho\right)=0,
\end{gathered}
$$

where terms of order $\hbar^{2}$ are neglected. Equation (A4a) is the Hamilton-Jacobi equation. Equation (A4b) is the 
continuity equation for evolution under a velocity field $p(q, t) / M$.

To interpret these equations, let the function $p(q, 0)$ describe a curve $\mathcal{A}_{0}$ in phase space, at an initial time $t=0$. If the wave function $\psi$ is represented as a sum of terms of the form given by Eq. (A1), then $p(q, 0)$ is multivalued, and the curve $\mathcal{A}_{0}$ has multiple branches, but for the moment we focus on a single branch. $\mathcal{A}_{0}$ is a Lagrangian manifold, determined by its generating function, $S(q, 0)$, via Eq. (A2). Now imagine that this curve carries a probability density whose projection onto the coordinate axis is $\rho(q, 0)$. It is convenient to picture a swarm of particles, sprinkled along $\mathcal{A}_{0}$ so that the fraction of particles between $q$ and $q+d q$ is given by $\rho(q, 0) d q$. From these initial conditions, each particle in the swarm evolves under Hamilton's equations, giving rise to a time-dependent curve $\mathcal{A}_{t}$ and density $\rho(q, t)$. The Hamilton-Jacobi equation governs the evolution of $\mathcal{A}_{t}$, through its generating function $S(q, t)$, and the continuity equation governs the evolution of the projected density $\rho(q, t)$.

With these considerations in mind, approximate solutions of the time-dependent Schrödinger equation are written in the form

$$
\psi(q, t)=\sum_{b} \sqrt{\rho_{b}(q, t)} \exp \left[\frac{i}{\hbar} S_{b}(q, t)-i \mu_{b} \frac{\pi}{2}\right] .
$$

The sum is taken over the branches of a Lagrangian manifold $\mathcal{A}_{t}$ whose generating function $S(q, t)$ satisfies the Hamilton-Jacobi equation [Eq. (A4a)], and the projected probability density for each branch satisfies the continuity equation [Eq. (A4b)].

The quantities $\mu_{b}$ are Maslov indices. These determine the relative quantum phases of the various branches of $\mathcal{A}_{t}$, and they differ from one another by integer values. (By convention, the overall phase of $\psi$ is adjusted so that the $\mu_{b}$ 's themselves are integers.) See Ref. [77] for a more detailed discussion of Maslov indices, as well as a careful treatment of caustic points, where two branches of $\mathcal{A}_{t}$ meet and the manifold becomes "vertical" in the $(q, p)$ plane.

At the semiclassical level of approximation, Eq. (A5) connects the evolution of a quantum wave function to that of a swarm of classical particles "surfing" on a Lagrangian manifold. This perspective provides a natural starting point for a comparison between quantum and classical work distributions.

As an important example of a WKB wave function, consider a manifold $\mathcal{A}_{0}$ that is a single energy shell $E$ of a fixed Hamiltonian $H(q, p)$, and consider a microcanonical distribution of initial conditions on this manifold. Under time evolution, neither the manifold nor the probability distribution changes, as each trajectory merely goes around the energy shell. The solution of Eq. (A4a) in this case is

$$
S_{b}(q, t)=S_{b}(q, 0)-E t
$$

hence, by Eq. (A5), the wave function simply acquires a phase $e^{-i E t / \hbar}$. Thus, in the semiclassical limit, energy eigenstates correspond to microcanonical ensembles. Indeed, Eq. (A5) in this case leads to the WKB approximation for energy eigenstates that is familiar from undergraduate textbooks on quantum mechanics, where it is typically derived in a different manner. Moreover, a proper treatment of the Maslov indices leads to the quantization condition $\oint p(q) d q=[m+(1 / 2)] h$.

\section{APPENDIX B: SEMICLASSICAL TRANSITION PROBABILITIES IN THE BOUNDARY AREA}

Expanding the function $\Delta S(q, E)$ defined by Eq. (60) to cubic order, we get

$$
\begin{aligned}
& \Delta S(q, E) \approx \Delta S_{c}(E)+\Delta S_{c}^{\prime}(E)\left(q-q_{c}\right) \\
& \quad+\frac{1}{2} \Delta S_{c}^{\prime \prime}(E)\left(q-q_{c}\right)^{2}+\frac{1}{6} \Delta S_{c}^{\prime \prime \prime}(E)\left(q-q_{c}\right)^{3},
\end{aligned}
$$

where $\Delta S_{c}(E)=\Delta S\left(q_{c}, E\right)$ and primes indicate derivatives with respect to $q$. Note that here we expand $\Delta S$ around the coalescence point $q=q_{c}$, where the surface $\mathcal{A}$ becomes tangent to the surface $\mathcal{B}$ (see Fig. 8). We now evaluate the integral $\int d q \exp (i \Delta S / \hbar)$, using Eq. (B1).

Let us first consider the integral

$$
\begin{aligned}
I & =\int_{-\infty}^{+\infty} d q \exp \left[\frac{i}{\hbar} f(q)\right], \\
f(q) & =\alpha_{0}+\alpha_{1} q+\frac{1}{2} \alpha_{2} q^{2}+\frac{1}{6} \alpha_{3} q^{3} .
\end{aligned}
$$

Rewriting $f(q)$ as

$$
f(q)=\beta_{0}+\beta_{1}(q-c)+\frac{1}{3} \beta_{3}(q-c)^{3},
$$

where

$$
\begin{aligned}
& c=-\frac{\alpha_{2}}{\alpha_{3}}, \quad \beta_{0}=\alpha_{0}-\frac{\alpha_{1} \alpha_{2}}{\alpha_{3}}+\frac{\alpha_{2}^{3}}{3 \alpha_{3}^{2}}, \\
& \beta_{1}=\alpha_{1}-\frac{\alpha_{2}^{2}}{2 \alpha_{3}}, \quad \beta_{3}=\frac{\alpha_{3}}{2},
\end{aligned}
$$

we obtain

$$
\begin{aligned}
I & =e^{i \beta_{0} / \hbar} \int_{-\infty}^{+\infty} d y \exp \left[\frac{i}{\hbar}\left(\beta_{1} y+\frac{\beta_{3}}{3} y^{3}\right)\right] \\
& =2 \pi e^{i \beta_{0} / \hbar}\left(\frac{\hbar}{\left|\beta_{3}\right|}\right)^{1 / 3} \operatorname{Ai}\left(\frac{\beta_{1}}{\hbar^{2 / 3} \beta_{3}^{1 / 3}}\right),
\end{aligned}
$$

using the integral representation of the Airy function, 


$$
\operatorname{Ai}(\zeta)=\frac{1}{2 \pi} \int_{-\infty}^{+\infty} d t \exp \left[i\left(\zeta t+\frac{t^{3}}{3}\right)\right]
$$

We now use this result to evaluate

$$
I(E)=\int d q \exp \left[\frac{i}{\hbar} \Delta S(q, E)\right]
$$

for small $\hbar$, applying the stationary phase approximation and treating $E$ as a parameter of the integral.

The cubic expansion for $\Delta S$ given by Eq. (B1) gives us the coefficients

$$
\begin{gathered}
\alpha_{0}(E)=\Delta S\left(q_{c}, E\right), \\
\alpha_{1}(E)=\Delta S^{\prime}\left(q_{c}, E\right)=\Delta p\left(q_{c}, E\right), \\
\alpha_{2}(E)=\Delta S^{\prime \prime}\left(q_{c}, E\right)=\Delta p^{\prime}\left(q_{c}, E\right), \\
\alpha_{3}(E)=\Delta S^{\prime \prime \prime}\left(q_{c}, E\right)=\Delta p^{\prime \prime}\left(q_{c}, E\right),
\end{gathered}
$$

with $\Delta p=p^{\mathcal{A}}-p^{\mathcal{B}}=\Delta S^{\prime}$ [Eq. (61)]. Note that

$$
\alpha_{1}\left(E_{c}\right)=\alpha_{2}\left(E_{c}\right)=0
$$

since $\mathcal{A}$ and $\mathcal{B}$ are tangent at $q_{c}$ when $E=E_{c}$. The integral $I(E)$ is now given by Eq. (B5), with $\beta_{0}, \beta_{1}$, and $\beta_{3}$ obtained from $\alpha_{0}, \alpha_{1}, \alpha_{2}$, and $\alpha_{3}$ via Eq. (B4). This result simplifies if we consider how the $\beta$ 's behave in the vicinity of $E=E_{c}$ as $\hbar \rightarrow 0$.

Let us define

$$
\begin{gathered}
p_{c}=p^{\mathcal{A}}\left(q_{c}\right)=p^{\mathcal{B}}\left(q_{c}, E_{c}\right), \\
p_{c}^{\prime}=\frac{\partial p^{\mathcal{A}}}{\partial q}\left(q_{c}\right)=\frac{\partial p^{\mathcal{B}}}{\partial q}\left(q_{c}, E_{c}\right), \\
k=\frac{\partial^{2} p^{\mathcal{A}}}{\partial q^{2}}\left(q_{c}\right)-\frac{\partial^{2} p^{\mathcal{B}}}{\partial q^{2}}\left(q_{c}, E_{c}\right)=\alpha_{3}\left(E_{c}\right), \\
\nu=\frac{\partial p^{\mathcal{B}}}{\partial E}\left(q_{c}, E_{c}\right),
\end{gathered}
$$

Now look at the argument of the Airy function in Eq. (B5),

$$
\zeta(E, \hbar)=\frac{\beta_{1}(E)}{\hbar^{2 / 3} \beta_{3}(E)^{1 / 3}},
$$

and consider a fixed range of $\zeta$ values, $\zeta_{-}<0<\zeta_{+}$, chosen so that the asymptotic approximations for the Airy function [Eq. (65)] are accurate at both $\zeta_{-}$and $\zeta_{+}$. Noting that $\zeta\left(E_{c}, \hbar\right)=0$ and expanding $\zeta(E, \hbar)$ to first order in $\left(E-E_{c}\right)$, we see that as $\hbar \rightarrow 0$, the fixed range $\left[\zeta_{-}, \zeta_{+}\right]$translates to a range of energies whose width scales like $\hbar^{2 / 3}$. Thus, we are interested in energies that have the following scaling relation:

$$
\epsilon \sim \hbar^{2 / 3},
$$

where $\epsilon=E-E_{c}$. To leading order in $\hbar$, and expressing quantities in terms of $\epsilon$ rather than $E$, we have

$\zeta=\frac{a \epsilon}{\hbar^{2 / 3} b^{1 / 3}}, \quad$ where $a=\frac{\partial \beta_{1}}{\partial \epsilon}(\epsilon=0)$,

$b=\beta_{3}(\epsilon=0)$.

Now recall that $\beta_{1}=\alpha_{1}-\alpha_{2}^{2} / 2 \alpha_{3}$. In the vicinity of $\epsilon=0$ (where $\alpha_{1}=\alpha_{2}=0$ ), we have $\alpha_{1} \sim \epsilon \sim \hbar^{2 / 3}$ and $\alpha_{2}^{2} / 2 \alpha_{3} \sim \epsilon^{2} \sim \hbar^{4 / 3}$; hence, the latter term can be ignored. We thus write

$$
a=\frac{\partial \alpha_{1}}{\partial \epsilon}(0)=-\nu, \quad b=\frac{1}{2} \alpha_{3}(0)=\frac{k}{2} ;
$$

therefore,

$$
\zeta=-\left(\frac{2}{k \hbar^{2}}\right)^{1 / 3} \nu \epsilon
$$

Combining results and discarding terms that vanish as $\hbar \rightarrow 0$, Eq. (B5) finally gives us

$$
I(\epsilon)=2 \pi\left(\frac{2 \hbar}{|k|}\right)^{1 / 3} \exp \left[\frac{i}{\hbar} \alpha_{0}(\epsilon)\right] \operatorname{Ai}\left(-\frac{2^{1 / 3} \nu \epsilon}{k^{1 / 3} \hbar^{2 / 3}}\right),
$$

which is equivalent to Eq. (62).

[1] C. Bustamante, J. Liphardt, and F. Ritort, The Nonequilibrium Thermodynamics of Small Systems, Phys. Today 58, No. 7, 43 (2005).

[2] C. Jarzynski, Equalities and Inequalities: Irreversibility and the Second Law of Thermodynamics at the Nanoscale, Annu. Rev. Condens. Matter Phys. 2, 329 (2011).

[3] U. Seifert, Stochastic Thermodynamics, Fluctuation Theorems and Molecular Machines, Rep. Prog. Phys. 75, 126001 (2012).

[4] Nonequilibrium Statistical Physics of Small Systems: Fluctuation Relations and Beyond, edited by R. Klages, W. Just, and C. Jarzynski (Wiley-VCH, Weinheim, 2013).

[5] B. Piechocinska, Information Erasure, Phys. Rev. A 61, 062314 (2000).

[6] J. Kurchan, A Quantum Fluctuation Theorem, arXiv:condmat/0007360v2.

[7] H. Tasaki, Jarzynski Relations for Quantum Systems and Some Applications, arXiv:cond-mat/0009244v2.

[8] S. Yukawa, A Quantum Analogue of the Jarzynski Equality, J. Phys. Soc. Jpn. 69, 2367 (2000). 
[9] S. Mukamel, Quantum Extension of the Jarzynski Relation: Analogy with Stochastic Dephasing, Phys. Rev. Lett. 90, 170604 (2003).

[10] T. Monnai and S. Tasaki, Quantum Correction of Fluctuation Theorem, arXiv:cond-mat/0308337.

[11] V. Chernyak and S. Mukamel, Effect of Quantum Collapse on the Distribution of Work in Driven Single Molecules, Phys. Rev. Lett. 93, 048302 (2004).

[12] W. De Roeck and C. Maes, Quantum Version of FreeEnergy-Irreversible-Work Relations, Phys. Rev. E 69, 026115 (2004).

[13] A. E. Allahverdyan and Th. M. Nieuwenhuizen, Fluctuations of Work from Quantum Subensembles: The Case against Quantum Work-Fluctuation Theorems, Phys. Rev. E 71, 066102 (2005).

[14] A. Engel and R Nolte, Jarzynski Equation for a Simple Quantum System: Comparing Two Definitions of Work, Europhys. Lett. 79, 10003 (2007).

[15] P. Talkner, E. Lutz, and P. Hänggi, Fluctuation Theorems: Work Is Not an Observable, Phys. Rev. E 75, 050102(R) (2007).

[16] J. Teifel and G. Mahler, Model Studies on the Quantum Jarzynski Relation, Phys. Rev. E 76, 051126 (2007).

[17] G. E. Crooks, On the Jarzynski Relation for Dissipative Quantum Dynamics, J. Stat. Mech. (2008) P10023.

[18] M. Campisi, P Talkner, and P Hänggi, Fluctuation Theorem for Arbitrary Open Quantum Systems, Phys. Rev. Lett. 102, 210401 (2009).

[19] M. Esposito, U. Harbola, and S. Mukamel, Nonequilibrium Fluctuations, Fluctuation Theorems, and Counting Statistics in Quantum Systems, Rev. Mod. Phys. 81, 1665 (2009).

[20] M. Campisi, P. Hänggi, and P. Talkner, Colloquium: Quantum Fluctuation Relations: Foundations and Applications, Rev. Mod. Phys. 83, 771 (2011).

[21] M. Campisi, P. Talkner, and P. Hänggi, Influence of Measurements on the Statistics of Work Performed on a Quantum System, Phys. Rev. E 83, 041114 (2011).

[22] J. M. Horowitz, Quantum-Trajectory Approach to the Stochastic Thermodynamics of a Forced Harmonic Oscillator, Phys. Rev. E 85, 031110 (2012).

[23] F. Liu, Derivation of Quantum Work Equalities Using a Quantum Feynman-Kac Formula, Phys. Rev. E 86, 010103 (2012).

[24] Y. Subasi and B. L. Hu, Quantum and Classical Fluctuation Theorems from a Decoherent Histories, Open-System Analysis, Phys. Rev. E 85, 011112 (2012).

[25] T. Albash, D. A. Lidar, M. Marvian, and P. Zanardi, Fluctuation Theorems for Quantum Processes, Phys. Rev. E 88, 032146 (2013).

[26] P. Talkner, M. Morillo, Y. Yi, and P. Hänggi, Statistics of Work and Fluctuation Theorems for Microcanonical Initial States, New J. Phys. 15, 095001 (2013).

[27] S. Deffner, Quantum Entropy Production in Phase Space, Europhys. Lett. 103, 30001 (2013).

[28] F. W. J. Hekking and J. P. Pekola, Quantum Jump Approach for Work and Dissipation in a Two-Level System, Phys. Rev. Lett. 111, 093602 (2013).

[29] P. Solinas, D. V. Averin, and J. P. Pekola, Work and Its Fluctuations in a Driven Quantum System, Phys. Rev. B 87, 060508(R) (2013).
[30] F. Liu, Equivalence of Two Bochkov-Kuzovlev Equalities in Quantum Two-Level Systems, Phys. Rev. E 89, 042122 (2014).

[31] F. Liu, Calculating Work in Adiabatic Two-Level Quantum Markovian Master Equations: A Characteristic Function Method, Phys. Rev. E 90, 032121 (2014).

[32] M. Silaev, T. T. Heikkilä, and P. Virtanen, LindbladEquation Approach for the Full Counting Statistics of Work and Heat in Driven Quantum Systems, Phys. Rev. E 90, 022103 (2014).

[33] J. Salmilehto, P. Solinas, and M. Möttönen, Quantum Driving and Work, Phys. Rev. E 89, 052128 (2014).

[34] A. E. Rastegin and K. Życzkowski, Jarzynski Equality for Quantum Stochastic Maps, Phys. Rev. E 89, 012127 (2014).

[35] G. Watanabe, B. Prasanna Venkatesh, P. Talkner, M. Campisi, and P. Hänggi, Quantum Fluctuation Theorems and Generalized Measurements during the Force Protocol, Phys. Rev. E 89, 032114 (2014).

[36] A. J. Roncaglia, F. Cerisola, and J. Pablo Paz, Work Measurement as a Generalized Quantum Measurement, Phys. Rev. Lett. 113, 250601 (2014).

[37] A. E. Allahverdyan, Nonequilibrium Quantum Fluctuations of Work, Phys. Rev. E 90, 032137 (2014).

[38] Z. Gong, S. Deffner, and H. T. Quan, Interference of Identical Particles and the Quantum Work Distribution, Phys. Rev. E 90, 062121 (2014).

[39] N. Y. Halpern, A. J. P. Garner, O. C. O. Dahlsten, and V. Vedral, Unification of Fluctuation Theorems and One-Shot Statistical Mechanics, arXiv:1409.3878v1.

[40] S. Suomela, P. Solinas, J. P. Pekola, J. Ankerhold, and T. Ala-Nissila, Moments of Work in the Two-Point Measurement Protocol for a Driven Open Quantum System, Phys. Rev. B 90, 094304 (2014).

[41] K. Viisanen, S. Suomela, S. Gasparinetti, O.-P. Saira, J. Ankerhold, and J. P. Pekola, Incomplete Measurement of Work in a Dissipative Two Level System, New J. Phys. 17, 055014 (2015).

[42] M. Lostaglio, D. Jennings, and T. Rudolph, Description of Quantum Coherence in Thermodynamic Processes Requires Constraints beyond Free Energy, Nat. Commun. 6, 6383 (2015).

[43] P. Hänggi and P. Talkner, The Other QFT, Nat. Phys. 11, 108 (2015).

[44] B. Prasanna Venkatesh, G. Watanabe, and P. Talkner, Quantum Fluctuation Theorems and Power Measurements, New J. Phys. 17, 075018 (2015).

[45] S. Suomela, J. Salmilehto, I. G. Savenko, T. Ala-Nissila, and M. Möttönen, Fluctuations of Work in Nearly Adiabatically Driven Open Quantum Systems, Phys. Rev. E 91, 022126 (2015).

[46] P. Solinas and S. Gasparinetti, Nonclassical Features in the Distribution of Work Performed on a Quantum System, arXiv:1504.01574v1.

[47] J. Goold, M. Huber, A. Riera, L. del Rio, and P. Skrzypzyk, The Role of Quantum Information in Thermodynamics-A Topical Review, arXiv:1505.07835v1.

[48] F. Liu, Calculating Work in Weakly Driving Quantum Master Equations: Backward and Forward Equations, arXiv:1506.08343. 
[49] C. Elouard, A. Auffèves, and M. Clusel, Stochastic Thermodynamics in the Quantum Regime, arXiv:1507.00312.

[50] J. J. Alonso, E. Lutz, and A. Romito, Thermodynamics of Weakly Measured Quantum Systems, arXiv:1508.00438.

[51] H. T. Quan and C. Jarzynski, Validity of Nonequilibrium Work Relations for the Rapidly Expanding Quantum Piston, Phys. Rev. E 85, 031102 (2012).

[52] J. v. Neumann, Mathematical Foundations of Quantum Mechanics (Princeton University Press, Princeton, NJ, 1955).

[53] G. N. Bochkov and Y.E. Kuzovlev, General Theory of Thermal Fluctuations in Nonlinear Systems, Sov. Phys. JETP 45, 125 (1977).

[54] C. Jarzynski, Nonequilibrium Equality for Free Energy Differences, Phys. Rev. Lett. 78, 2690 (1997).

[55] G. E. Crooks, Entropy Production Fluctuation Theorem and the Nonequilibrium Work Relation for Free Energy Differences, Phys. Rev. E 60, 2721 (1999).

[56] G. Hummer and A. Szabo, Free Energy Reconstruction from Nonequilibrium Single-Molecule Pulling Experiments, Proc. Natl. Acad. Sci. U.S.A. 98, 3658 (2001).

[57] J. Liphardt, S. Dumont, S. P. Smith, I. Tinoco, Jr, and C. Bustamante, Equilibrium Information from Nonequilibrium Measurements in an Experimental Test of Jarzynskis Equality, Science 296, 1832 (2002).

[58] D. Collin, F. Ritort, C. Jarzynski, S. B. Smith, I. Tinoco, Jr, and C. Bustamante, Verification of the Crooks Fluctuation Theorem and Recovery of RNA Folding Free Energies, Nature (London) 437, 231 (2005).

[59] G. Huber, F. Schmidt-Kaler, S. Deffner, and E. Lutz, Employing Trapped Cold Ions to Verify the Quantum Jarzynski Equality, Phys. Rev. Lett. 101, 070403 (2008).

[60] R. Dorner, S. R. Clark, L. Heaney, R. Fazio, J. Goold, and V. Vedral, Extracting Quantum Work Statistics and Fluctuation Theorems by Single-Qubit Interferometry, Phys. Rev. Lett. 110, 230601 (2013).

[61] L. Mazzola, G. De Chiara, and M. Paternostro, Measuring the Characteristic Function of the Work Distribution, Phys. Rev. Lett. 110, 230602 (2013).

[62] M. Campisi, R. Blattmann, S. Kohler, D. Zueco, and P. Hänggi, Employing Circuit QED to Measure NonEquilibrium Work Fluctuations, New J. Phys. 15, 105028 (2013).

[63] T. B. Batalhão, A. M. Souza, L. Mazzola, R. Auccaise, R. S. Sarthour, I. S. Oliveira, J. Goold, G. De Chiara, M. Paternostro, and R. M. Serra, Experimental Reconstruction of Work Distribution and Study of Fluctuation Relations in a Closed Quantum System, Phys. Rev. Lett. 113, 140601 (2014).

[64] S. An, J.-N. Zhang, M. Um, D. Lv, Y. Lu, J. Zhang, Z.-Q. Yin, H. T. Quan, and K. Kim, Experimental Test of the Quantum Jarzynski Equality with a Trapped-Ion System, Nat. Phys. 11, 193 (2015).

[65] R. A. Marcus, Extension of the WKB Method to Wave Functinons and Transition Probability Amplitudes (S-Matrix) for Inelastic or Reactive Collisions, Chem. Phys. Lett. 7, 525 (1970).

[66] R. A. Marcus, Theory of Semiclassical Transition Probabilities(S-Matrix) for Inelastic and Reactive Collisions, J. Chem. Phys. 54, 3965 (1971).
[67] W. H. Miller, Classical-Limit Quantum Mechanics and the Theory of Molecular Collisions, Adv. Chem. Phys. 25, 69 (1974).

[68] C. D. Schwieters and J. B. Delos, Semiclassical Treatment of a Half-Cycle Pulse Acting on a One-Dimensional Rydberg Atom, Phys. Rev. A 51, 1023 (1995).

[69] C. D. Schwieters and J. B. Delos, Half-Cycle Pulse Acting on a One-Dimensional Rydberg Atom: Semiclassical Transition Amplitudes in Action and Angle Variables, Phys. Rev. A 51, 1030 (1995).

[70] S. Deffner and E. Lutz, Nonequilibrium Work Distribution of a Quantum Harmonic Oscillator, Phys. Rev. E 77, 021128 (2008).

[71] S. Deffner, O. Abah, and E. Lutz, Quantum Work Statistics of Linear and Nonlinear Parametric Oscillators, Chem. Phys. 375, 200 (2010).

[72] P. Talkner, P. S. Burada, and P. Hänggi, Statistics of Work Performed on a Forced Quantum Oscillator, Phys. Rev. E 78, 011115 (2008).

[73] M. Campisi, Increase of Boltzmann Entropy in a Quantum Forced Harmonic Oscillator, Phys. Rev. E 78, 051123 (2008).

[74] I. J. Ford, D. S. Minor, and S. J. Binnie, Symmetries of Cyclic Work Distributions for an Isolated Harmonic Oscillator, Eur. J. Phys. 33, 1789 (2012).

[75] K. Husimi, Miscellanea in Elementary Quantum Mechanics. 2, Prog. Theor. Phys. 9, 381 (1953).

[76] J.B. Delos, Semiclassical Calculation of Quantum Mechanical Wavefunctions, Adv. Chem. Phys. 65, 161 (1986).

[77] R. G. Littlejohn, The Van Vleck Formula, Maslov Theory, and Phase Space Geometry, J. Stat. Phys. 68, 7 (1992).

[78] R. P. Feynman and A. R. Hibbs, Quantum Mechanics and Path Integrals (McGraw-Hill, New York, 1965).

[79] M. V. Berry, Semiclassical Theory of Spectral Rigidity, Proc. R. Soc. A 400, 229 (1985).

[80] E. Doron, U. Smilansky, and A. Frenkel, Chaotic Scattering and Transmission Fluctuations, Physica (Amsterdam) 50D, 367 (1991).

[81] H. U. Baranger, R. A. Jalabert, and A. D. Stone, Weak Localization and Integrability in Ballistic Cavities, Phys. Rev. Lett. 70, 3876 (1993).

[82] D. J. Griffiths, Introduction to Quantum Mechanics (Prentice-Hall, Englewood Cliffis, NJ, 1995).

[83] L. I. Schiff, Quantum Mechanics (McGraw-Hill Book Company, New York, 1968).

[84] M. V. Berry, in Chaotic Behavior of Deterministic Systems, Proceedings of the Les Houches Summer School, Session XXXVI, edited by G. Iooss, R. H. Helleman, and R. Stora (North-Holland, Amsterdam, 1983), pp. 172-271.

[85] J.H. Van Vleck, The Correspondence Principle in the Statistical Interpretation of Quantum Mechanics, Proc. Natl. Acad. Sci. U.S.A. 14, 178 (1928).

[86] M. V. Berry, Semi-classical Mechanics in Phase Space: A Study of Wigners Function, Phil. Trans. R. Soc. A 287, 237 (1977).

[87] M. V. Berry, Evolution of Semiclassical Quantum States in Phase Space, J. Phys. A 12, 625 (1979).

[88] M. V. Berry, Regular and Irregular Semiclassical Wavefunctions, J. Phys. A 10, 2083 (1977). 